\title{
Statistical properties of mechanically generated surface gravity waves: a laboratory experiment in a three-dimensional wave basin
}

\author{
M. ONORATO ${ }^{1}$, L. CAVALERI $I^{2}$, S. FOUQUES \\ O. GRAMSTAD ${ }^{4}$, P. A. E. M. JANSSEN ${ }^{5}$, J. MONBALI U \\ A. R. OSBORNE ${ }^{1}$, C. PAKOZDI ${ }^{3}, M \cdot S E R I O O^{1}$, \\ C. T. STANSBERG ${ }^{3}$, A. TOFFOLI AND K. TRULSEN \\ ${ }^{1}$ Dipartimento di Fisica Generale, Università di Torino, Via P. Giuria 1, Torino, Italy \\ ${ }^{2}$ ISMAR, Castello 1364/A, Venezia, Italy \\ ${ }^{3}$ Norwegian Marine Technology Research Institute A.S. (MARINTEK), PO Box 4125, Valentinlyst, \\ Trondheim, Norway \\ ${ }^{4}$ Department of Mathematics, University of Oslo, PO Box 1053, Blindern, Oslo, Norway \\ ${ }^{5}$ ECMWF, Shinfield Park, Reading, UK \\ ${ }^{6}$ K.U. Leuven, Kasteelpark Arenberg 40, Heverlee, Belgium \\ ${ }^{7}$ Det Norske Veritas, Veritasveien 1, Høvik, Norway
}

(Received 15 April 2008 and in revised form 12 December 2008)

A wave basin experiment has been performed in the MARINTEK laboratories, in one of the largest existing three-dimensional wave tanks in the world. The aim of the experiment is to investigate the effects of directional energy distribution on the statistical properties of surface gravity waves. Different degrees of directionality have been considered, starting from long-crested waves up to directional distributions with a spread of $\pm 30^{\circ}$ at the spectral peak. Particular attention is given to the tails of the distribution function of the surface elevation, wave heights and wave crests. Comparison with a simplified model based on second-order theory is reported. The results show that for long-crested, steep and narrow-banded waves, the second-order theory underestimates the probability of occurrence of large waves. As directional effects are included, the departure from second-order theory becomes less accentuated and the surface elevation is characterized by weak deviations from Gaussian statistics.

\section{Introduction}

An important task in the study of surface gravity waves is the determination of the probability density function of the surface elevation. The knowledge of the probability of occurrence of large amplitude waves is essential for different engineering purposes such as the prediction of wave forces and structural responses or the design of offshore structures. A deep comprehension of the physical mechanisms of generation of such waves is a first step towards the development of an operational methodology for forecasting freak waves. Theoretically speaking, the analytical determination of the probability density function is not an easy task: it is well known that surface gravity waves obey nonlinear equations and, nowadays, a universal tool suitable for deriving the probability distribution function of a nonlinear system has not been developed yet. Fortunately, water waves are on average weakly nonlinear and solutions can be 
generally written as power series, where the small parameter, in the case of deep water waves, is the wave steepness $\varepsilon$. At the lowest order of approximation the water wave problem is linear and the solution can be written as a superposition of sinusoidal waves with random phases. The amplitudes of the wave components are related to the energy distribution in frequency and angle, i.e. the wave spectrum. According to the central limit theorem, the surface elevation is Normally distributed and the envelope is distributed according to the Rayleigh distribution.

In nature, however, waves tend to behave differently; crests are higher and troughs are shallower than predicted by linear theory. Higher order terms are then needed in order to take these effects into account. The solution of the water wave problem to second order in steepness was given by Hasselmann (1962) for arbitrary depth, and in Longuet-Higgins (1963) for deep water. The solution is just a generalization of the second-order Stokes expansion. The amplitudes of the second-order contribution are proportional to the square of the amplitudes of the first-order solution. Note that at this order of nonlinearity there is no evolution in time of the wave spectrum. The second-order contribution affects the probability of wave crests but not of wave heights. An analytical description of the probability density function of the surface elevation resulting from the second-order description is not an easy task. Nevertheless, under some further approximations, some results can be achieved. For example, Tayfun (1980) derived a distribution for wave crests under the hypothesis of unidirectional waves and narrow banded spectra (see also Tayfun \& Fedele 2007, for a description of different models). An interesting parametrization of the probability density function for wave crests, which fits well the output of the simulations of the second-order model and many experimental data, has been given by Forristall (2000).

At third order in wave steepness, there is a substantial change in the description of water waves: while bound modes are still present, resonances (or quasi-resonances) are also possible and, as a consequence, wave amplitudes (free waves) may change on a time scale of the order of $1 / \varepsilon^{2}$ wave periods. Therefore, at third order the probability density function of surface elevation may depend on the dynamics of the waves. As a matter of fact, numerical simulations of the nonlinear Schrödinger equation (Onorato et al. 2001) have shown that, for unidirectional waves, the formation of extreme waves (the tail of the probability density function) depends on the ratio between the wave steepness and the spectral bandwidth. This ratio has been named in Janssen (2003) the Benjamin-Feir index, BFI (see also Alber 1978). Indeed, a mechanism which is responsible for the formation of extreme waves is basically a generalization of the Benjamin-Feir instability (Benjamin \& Feir 1967) or modulational instability (Zakharov 1968), which formally applies only to a Stokes wave and a small perturbation. The relevance of the third-order nonlinearity on the occurrence of extreme waves in random spectra was already noted in unidirectional numerical simulations of the Euler equations by Brandini (2001) and Mori \& Yasuda (2002) and suspected some years before by Trulsen \& Dysthe (1997). Experimentally speaking, there has been a number of papers in which the role of the modulational instability on the statistical properties of long-crested surface gravity waves was recognized (Stansberg 1992; Onorato et al. 2004, 2005, 2006b). Concerning the determination of the probability density function of wave heights, Tayfun \& Lo (1990) (see also Mori \& Janssen 2006) extended the Rayleigh distribution by also including the contribution of the fourth-order moment (kurtosis); analytical results were then compared favourably with experimental data given in Mori et al. (2007). All the aforementioned results on third-order effects deal with long-crested waves. 
Interestingly enough, already a few years earlier, using numerical simulations of a modified Schrödinger equation (Dysthe equation, Dysthe 1979) in two horizontal dimensions, Onorato, Osborne \& Serio (2002a) noted that the number of extreme wave events was reduced when the directional spreading of the initial spectrum was increased. These results were then confirmed by Socquet-Juglard et al. (2005) who also reported numerical results using a modified Dysthe equation. For shortcrested waves they found deviation from Gaussian statistics only due to bound wave contributions, while free waves preserve Gaussian statistics despite third-order nonlinear evolution. The statistics of the numerically simulated sea surface elevation thus showed good agreement with the Tayfun (1980) distributions for directional spectra while they also confirmed that, in the case of long-crested waves, the secondorder model underpredicted notably the probability of occurrence of extreme waves. Indeed, they found that free waves do deviate from Gaussian statistics due to the modulational instability process. A detailed analysis on the effect of directionality was recently given by Gramstad \& Trulsen (2007). They performed a large number of simulations to reveal how the occurrence of freak waves in deep water depends on the group and crest lengths for fixed steepness. They found that there is a sharp qualitative transition between short- and long-crested seas, for a crest length of approximately 10 wavelengths. For short crest lengths the statistics of extreme waves deviates slightly from Gaussian and their occurrence is independent of group length. As expected, for long-crested lengths, the statistics of waves is strongly non-Gaussian.

A first experimental investigation on the effects of directionality on the fourth-order moment of the surface elevation was already performed by Stansberg (1994). More recent experimental results have been reported by Waseda (2006), who performed wave tank experiments using directional JONSWAP spectra. He found that the occurrence of extreme waves is significantly reduced when the directionality broadens. His experiments were performed at the University of Tokyo in a facility $50 \mathrm{~m}$ long, $10 \mathrm{~m}$ wide and $5 \mathrm{~m}$ deep with a segmented plunger-type directional wavemaker (32 plungers). Experimental results in a wave basin have also been performed by Denissenko, Lukaschuk \& Nazarenko (2007). The size of the tank used was $12 \mathrm{~m} \times 6 \mathrm{~m} \times 1.5 \mathrm{~m}$ with a wavemaker characterized by eight pistons. They performed experiments using a continuous bi-chromatic forcing and letting the walls of the tank reflect the waves in order to reach a quasi-isotropic case. Their conditions can be considered as characterized by large directional spreading; their analysis showed that the wave crests statistics was consistent with the Tayfun distribution.

In the present paper, our purpose is to study in detail the transition region between the strongly non-Gaussian behaviour of the surface elevation that characterizes the long-crested waves and the weakly non-Gaussian statistics that is typical of shortcrested seas. We want to confirm qualitatively, at least for the time being (a direct comparison of the data with numerical simulation will follow in a different paper), the numerical results obtained by Gramstad \& Trulsen (2007) and the experimental results obtained in a smaller scale experiment by Waseda (2006). Note that the width of the tank used by Waseda (2006) is only $10 \mathrm{~m}$ and in principle the results could be strongly affected by the reflection on lateral walls, especially when waves travel at a large angle with respect to the main direction of propagation.

The present paper is organized as follows. In $\S 2$ we describe the facilities that have been used for the experiments. We want to stress here that the experiments have been performed in one of the largest existing wave basin in the world, equipped with a new multi-flap generator composed of 144 flaps. A description of the different wave fields considered for the experiments is also given. The resulting statistical properties 

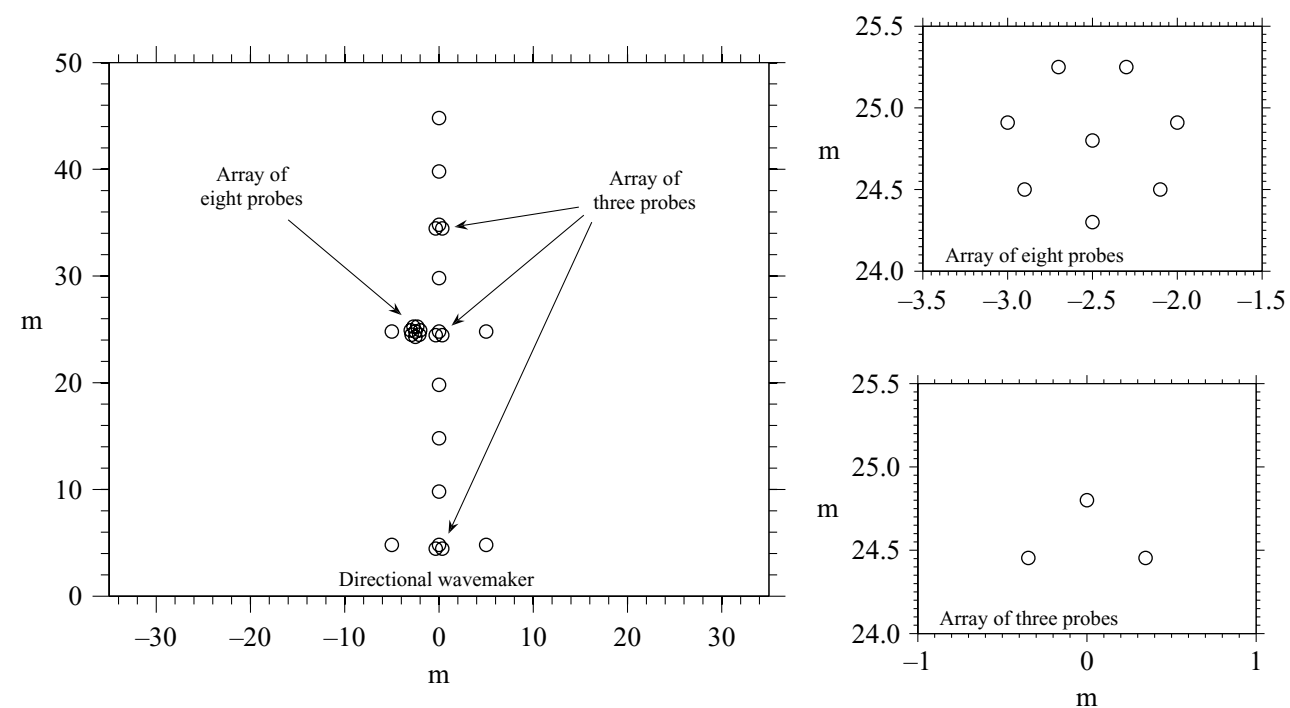

FIGURE 1. Wave basin and position of the wave gauges.

of the surface elevation, wave heights, wave crests are reported in $\S 3$. A discussion of the results and conclusions are then included in $\S 4$.

\section{The wave basin at MARINTEK}

The experiments have been performed at the MARINTEK wave facilities in Trondheim, Norway. Waves have been generated in a large rectangular wave basin. The Ocean Laboratory (see figure 1), as the MARINTEK three-dimensional wave tank is referred to, has dimensions $70 \mathrm{~m} \times 50 \mathrm{~m}$. The basin is equipped with a system that is capable of changing the water depth: the bottom of the basin moves up or down in order to achieve the desired uniform depth. The maximum depth is $10 \mathrm{~m}$, uniform throughout the basin. For the present experiment the water depth was fixed at $3 \mathrm{~m}$. The basin is fitted with two sets of wavemakers. Along the $50 \mathrm{~m}$ side there is a double flap, hydraulically operated unit for generating long-crested, regular and irregular waves. The second wavemaker (the one we have used for our present tests) is fitted along the $70 \mathrm{~m}$ side of the basin. It consists of altogether 144 individually computer-controlled flaps. This unit can generate short-crested seas within a wide range of directional distributions of the wave energy. Regular and irregular waves, with a desired angular and frequency distribution, can be generated. Each of the 144 flaps is $0.434 \mathrm{~m}$ wide and hinged at a depth of $1.02 \mathrm{~m}$ below the water surface. Each flap is individually controlled. In order to reduce wave reflections, the wave basin is equipped with an absorbing sloping beach at one side (opposite to the wavemaker); reflections in amplitude have been estimated to be less than $5 \%$ after 30 min of irregular waves of peak period of $1 \mathrm{~s}$.

Wave measurements have been concentrated along the central axis of the basin (see figure 1). Wave probes, which are held across the water surface by tripods laying on the bottom, are at $5 \mathrm{~m}$ intervals; the surface elevation is recorded with a sampling frequency of $80 \mathrm{~Hz}$. At locations 5, 25 and $35 \mathrm{~m}$ from the wavemaker, besides the single probe, two additional probes were deployed to allow the reconstruction of 


$\begin{array}{lcccccc}\text { Experiment } & T_{p}(\mathrm{~s}) & \alpha & \gamma & H_{s}(\mathrm{~m}) & k_{p} H_{s} / 2 & \mathrm{BFI} \\ \mathrm{A} & 1.0 & 0.014 & 3.0 & 0.06 & 0.13 & 0.70 \\ \mathrm{~B} & 1.0 & 0.016 & 6.0 & 0.08 & 0.16 & 1.10\end{array}$

TABLE 1. Imposed parameters in the experiments.

directional properties. At $25 \mathrm{~m}$ from the wavemaker, moreover, an eight-gauge array, which was arranged as a regular heptagon plus a central probe, was used to gather more detailed directional spectra (see, for example, Young 1994, for a discussion on the reconstruction of directional spectra from an $n$-gauge array).

\subsection{The conditions at the wavemaker}

In the present paper only irregular waves are described, even though a large number of experiments with monochromatic waves have been performed in order to test the facility. Complex Fourier amplitudes are generated, each with its modulus, randomly chosen from a Rayleigh distribution around the 'target' spectrum. The phases are randomly chosen from a uniform distribution between 0 and $2 \pi$. For the generation of input wave spectra the JONSWAP formulation (see, e.g. Komen et al. 1994) has been used to model the wave energy in the frequency domain:

$$
F(\omega)=\frac{\alpha g^{2}}{\omega^{5}} \exp \left[-\frac{5}{4}\left(\frac{\omega}{\omega_{p}}\right)^{-4}\right] \gamma^{\exp \left(\omega-\omega_{p}\right)^{2} / 2 \sigma_{j}^{2} \omega_{p}^{2}} .
$$

We have chosen to describe the wave field with a peak period $T_{p}=1 \mathrm{~s}$, which corresponds to a dominant wavelength of $1.56 \mathrm{~m}$. We should mention that, considering the water depth of $3 \mathrm{~m}$, waves have been generated in finite water depth $\left(k_{p} h \approx 12.1\right)$, but deep enough to include the modulational instability process (see, for example, Janssen \& Onorato 2007), at least for quasi-monochromatic waves. Two different types of experiments $\mathrm{A}$ and $\mathrm{B}$ characterized by two values of the Phillips parameter $\alpha$ and the peak enhancement factor $\gamma$ have been considered then. The values of the input (imposed) spectral parameters, the significant wave height, the wave steepness and BFI of the two experiments $\mathrm{A}$ and $\mathrm{B}$ are summarized in table 1 . Herein, the BFI is calculated as the ratio of the wave steepness $k_{p} H_{s} / 2$ to the spectral bandwidth $\Delta k / k_{p}$, where $\Delta k$ is a measure of the width of the spectrum estimated as the half-width at the half-maximum (see Onorato et al. 2006b, for details). It should be mentioned that in Socquet-Juglard et al. (2005) and Gramstad \& Trulsen (2007) the BFI is $\sqrt{2}$ times smaller than the one above defined because they estimate amplitudes as $H_{s} /(2 \sqrt{2})$.

A $\cos ^{N}\left(\theta-\theta_{m}\right)$ function is then applied to model the energy in the directional domain (see, for example, Hauser et al. 2005, for a review). In order to consider different degrees of directional spreading, different values of the spreading coefficient $N$ have been used, ranging from fairly long-crested (large $N)$ to short-crested ( $\operatorname{small} N$ ) waves. The following values have been selected: $N=840,200,90,50,24$. In figure 2 we show the directional distribution from the analytical formula; in the same plot, just for reference, we also include the $\operatorname{sech}^{2}$ parametrization described in Komen et al. (1994). For comparison, we have performed a number of experiments to simulate unidirectional waves too.

In order to have enough waves to perform a significant statistical analysis, four realizations of the random sea surface from a given spectrum have been performed by using different sets of random amplitudes and phases; for each test, $20 \mathrm{~min}$ of 


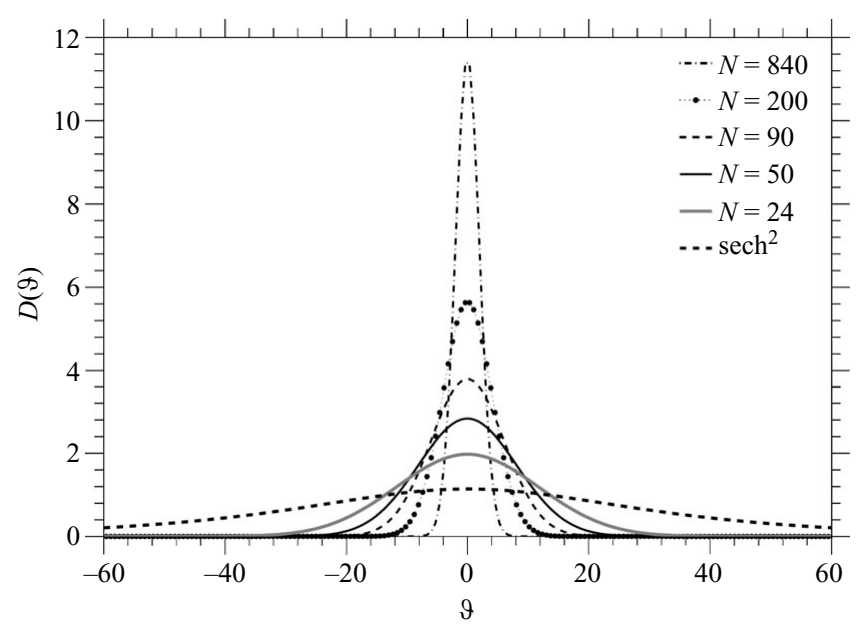

FIGURE 2. Analytical form of the directional distribution as a function of angle $\theta$ for different values of the parameter $N$; the $\operatorname{sech}^{2}$ distribution is included for comparison.

wave records were collected, including ramp-up time. For the present tests we have analysed the signals after $3 \mathrm{~min}$, thus there are $17 \mathrm{~min}$ left which we would use for processing. In these tests, there is no second-order correction of input signals.

\section{Experimental results}

\subsection{Significant wave height and wave spectra}

Here, we describe some properties of the wave field such as the significant wave height and the energy wave spectrum along the wave basin. In figure 3 , we present the significant wave height, calculated as four times the standard deviation $\sigma$ as a function of the distance from the wavemaker $x$ divided by the wavelength corresponding to the peak of the spectrum at the first probe $L$. It is evident that the wave energy slightly oscillates as waves start propagating. The observed fluctuation are of the order of $5 \%$ and $7 \%$ for experiments $\mathrm{A}$ and $\mathrm{B}$, respectively. As the waves propagate towards the end of the basin, the wave energy shows a decreasing trend. This reduction is almost negligible for experiment $\mathrm{A}(<5 \%$ of the value at the first probe), while it is more significant for experiment $\mathrm{B}$ as it reaches $9 \%$ of the significant wave height at the first probe. We should mention that the present experiment was not designed to study wave breaking and hence no quantitative measures of the wave breaking are available. Nonetheless, we can confidently state that wave breaking was observed more frequently in experiment B. This is therefore consistent with the observed loss of energy, especially for large BFI (see also Onorato et al. 2006b).

In figures 4 and 5 , we show the evolution of the frequency wave spectrum at three different non-dimensional distances from the wavemaker: $x / L=3.1 ; x / L=15.9$; $x / L=28.7$. Note that the frequency spectrum is measured as Fourier transform of the recorded time series at one single probe and not as the integration over the directions of a two-dimensional spectrum. Similarly to previous flume experiments (Onorato et al. 2006b), we observed that in all cases the level of the tail of the frequency spectrum is reduced as the waves propagate along the basin. Moreover, a downshift of the spectral peak is visible at $x / L=28.7$ regardless of the directional 

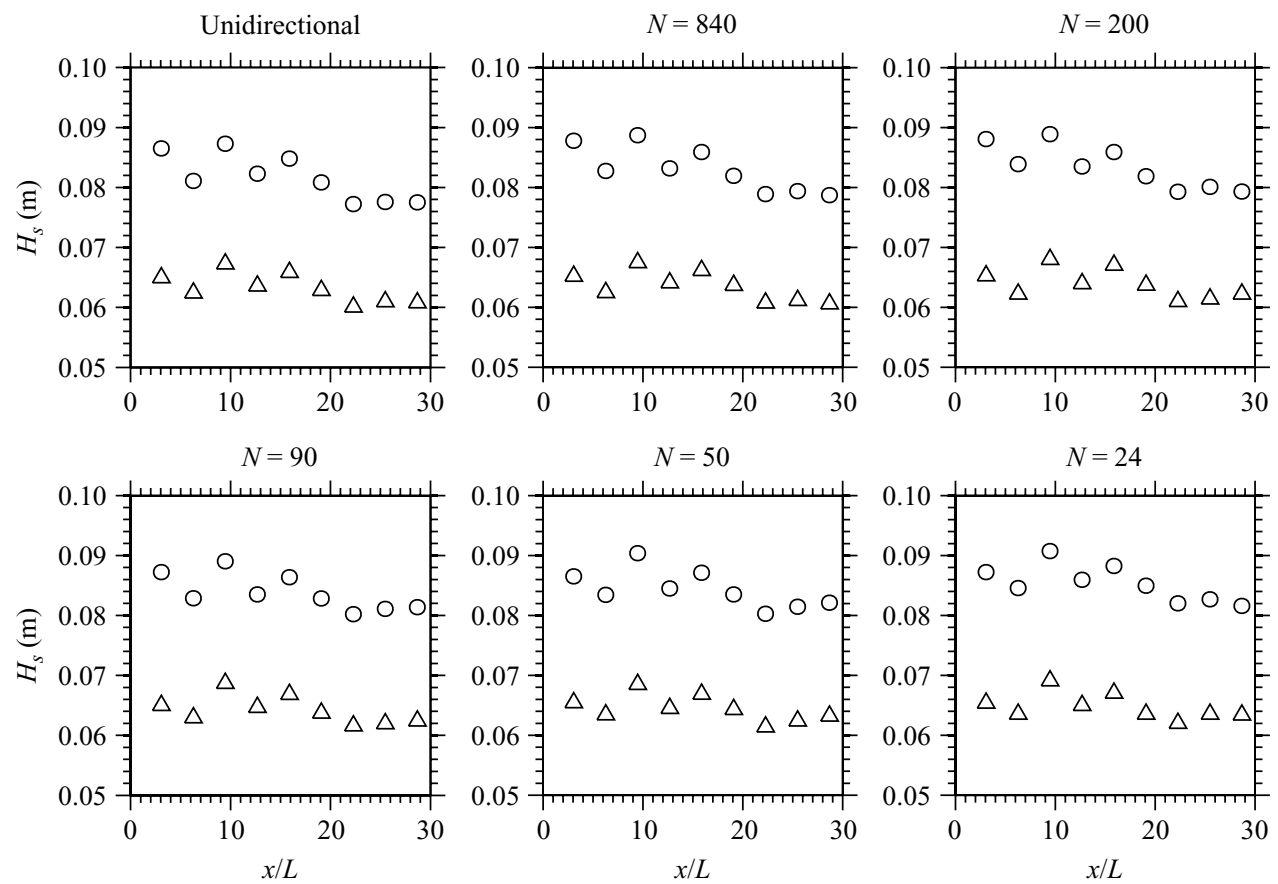

FIGURE 3. Significant wave height as a function of the non-dimensional distance from the wavemaker: experiment $A(\triangle)$, experiment $B(o)$.

properties of the wave field; this feature is especially evident for the experiment B (figure 5). The finding is consistent with numerical simulations in Dysthe et al. (2003), where a change of the spectrum is already observed on the scale of the Benjamin-Feir instability (see also Onorato et al. 2002b). In some spectra, for large $N$, there is also an indication of a second harmonic.

Also the directional properties of the wave spectrum are expected to change as waves start propagating from the wavemaker. A qualitative description of the directional distribution was obtained at locations where arrays of wave gauges were deployed; the wavelet directional method (Donelan, Drennan \& Magnusson 1996) was used to this end. The selected method provides a rather good description of wave directionality (see Donelan et al. 1996, for a detailed analysis of the method and comparison with the maximum likelihood method). In figure 6 , we present a few examples of directional wave spectra as recorded near the wavemaker $(x / L=3.1)$ and in the middle of the wave basin $(x / L=15.9)$ for initially long-crested $(N=840)$ and short-crested $(N=24)$ wave fields. It is evident that the wave spectrum becomes broader as waves evolve along the basin; changes in the directional distribution were observed for all selected sea states. This result is qualitatively consistent with numerical simulations of Schrödinger-type equations performed in Dysthe et al. (2003) and Socquet-Juglard et al. (2005).

\subsection{Higher order statistics: skewness and kurtosis}

We now investigate some statistical properties of the surface elevation. In figures 7 and 8 , we present the skewness and kurtosis as a function of the non-dimensional distance from the wavemaker. Whereas the first describes the vertical asymmetry of 

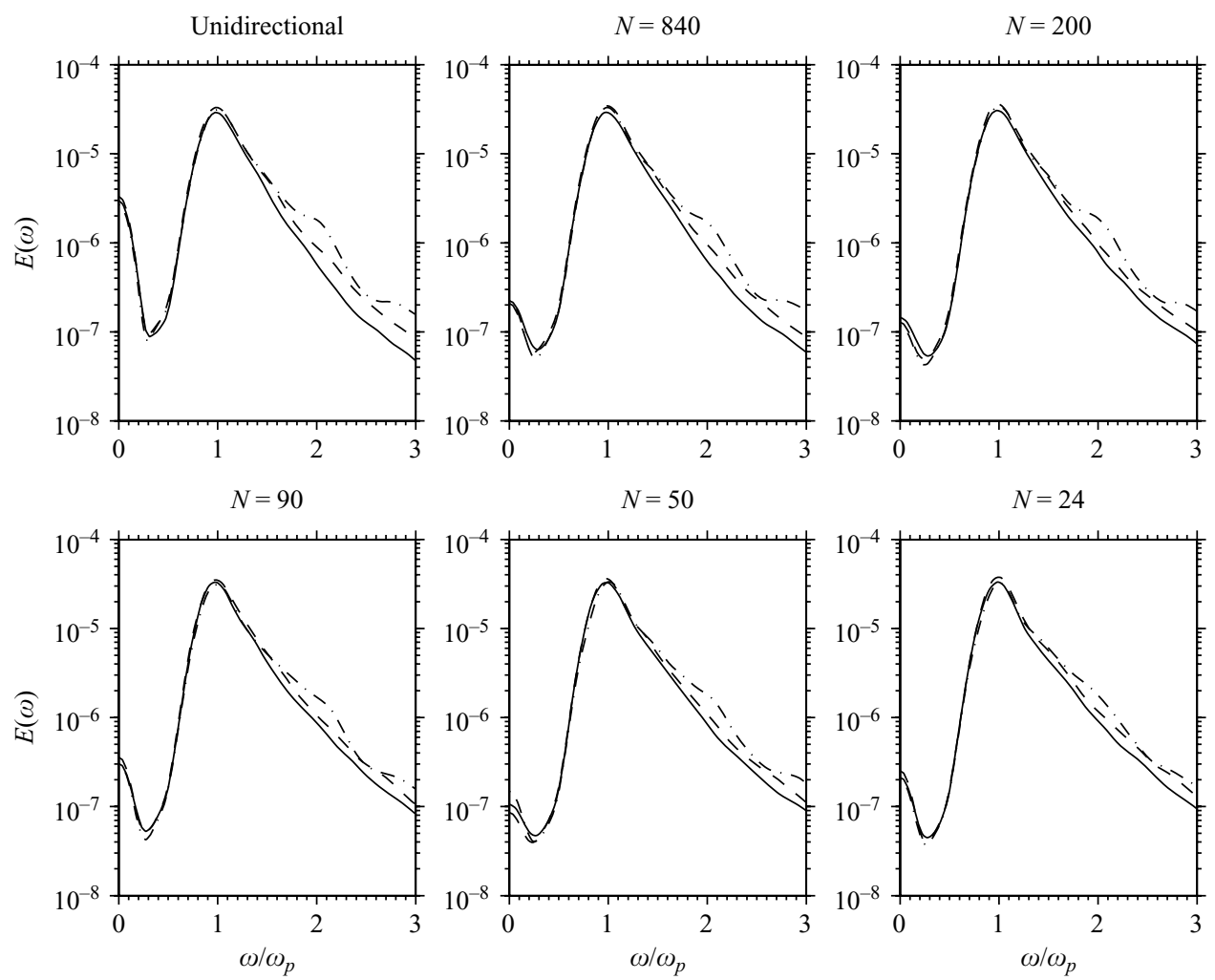

Figure 4. Frequency wave spectra for experiment A: $x / L=3.1$ (dash-dotted line); $x / L=15.9$ (dashed line); $x / L=28.7$ (solid line).

the wave profile, the latter provides an indication about the presence of extreme waves in the time series. For Gaussian (linear) processes, the skewness and kurtosis assume the value of 0 and 3 , respectively.

Traditionally, departures from the Gaussian statistics have been attributed to the presence of bound waves. In order to give an estimate of the skewness and kurtosis, we adopt the narrow-banded approximation of the second-order theory (LonguetHiggins 1963). Under such hypothesis, for deep water waves, the skewness $\left(\lambda_{3}\right)$ and kurtosis $\left(\lambda_{4}\right)$ take the following form:

$$
\begin{gathered}
\lambda_{3}=3 k_{p} \sigma, \\
\lambda_{4}=3+24\left(k_{p} \sigma\right)^{2},
\end{gathered}
$$

where $k_{p}$ is the wavenumber at the spectral peak and $\sigma$ is the standard deviation. In figures 7 and 8 , the values of the skewness and kurtosis along the wave basin are presented as calculated by (3.1) and (3.2); $k_{p}$ and $\sigma$ are estimated at each probe. We mention that the second-order theory has been derived from the Euler equations as a superposition of linear travelling wave solutions with random phases corrected to second-order. Nonlinear four-wave resonant and quasi-resonant interactions are not included; therefore, the spectrum does not change in time as the wave field evolves. Equations (3.1) and (3.2) are usually a decent approximation of the third- and the fourth-order moments of the second-order theory for long-crested waves; however, for waves with directional spreading, they tend to overpredict the value of the skewness 

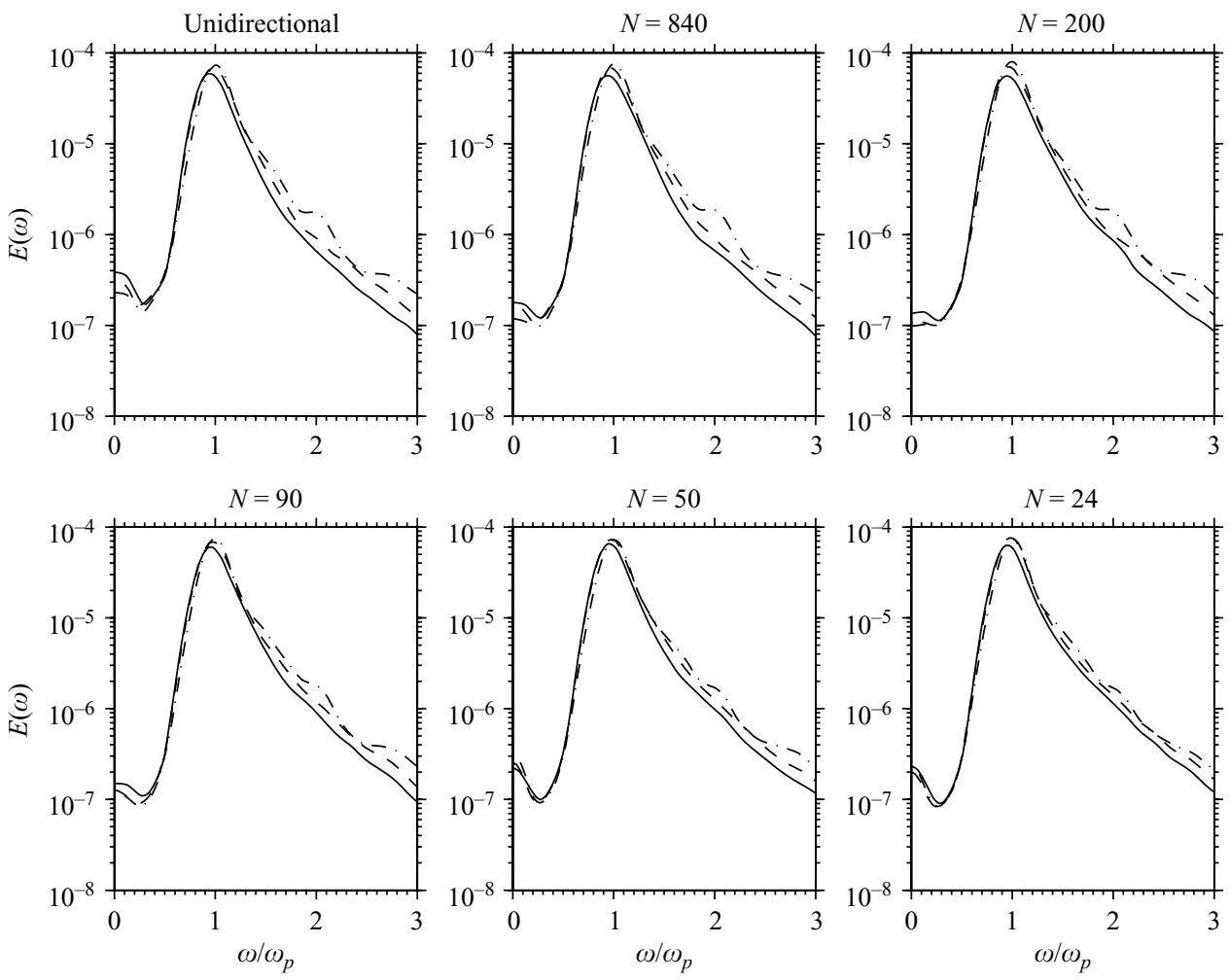

FIGURE 5. Frequency wave spectra for experiment B: $x / L=3.1$ (dash-dotted line); $x / L=15.9$ (dashed line); $x / L=28.7$ (solid line).
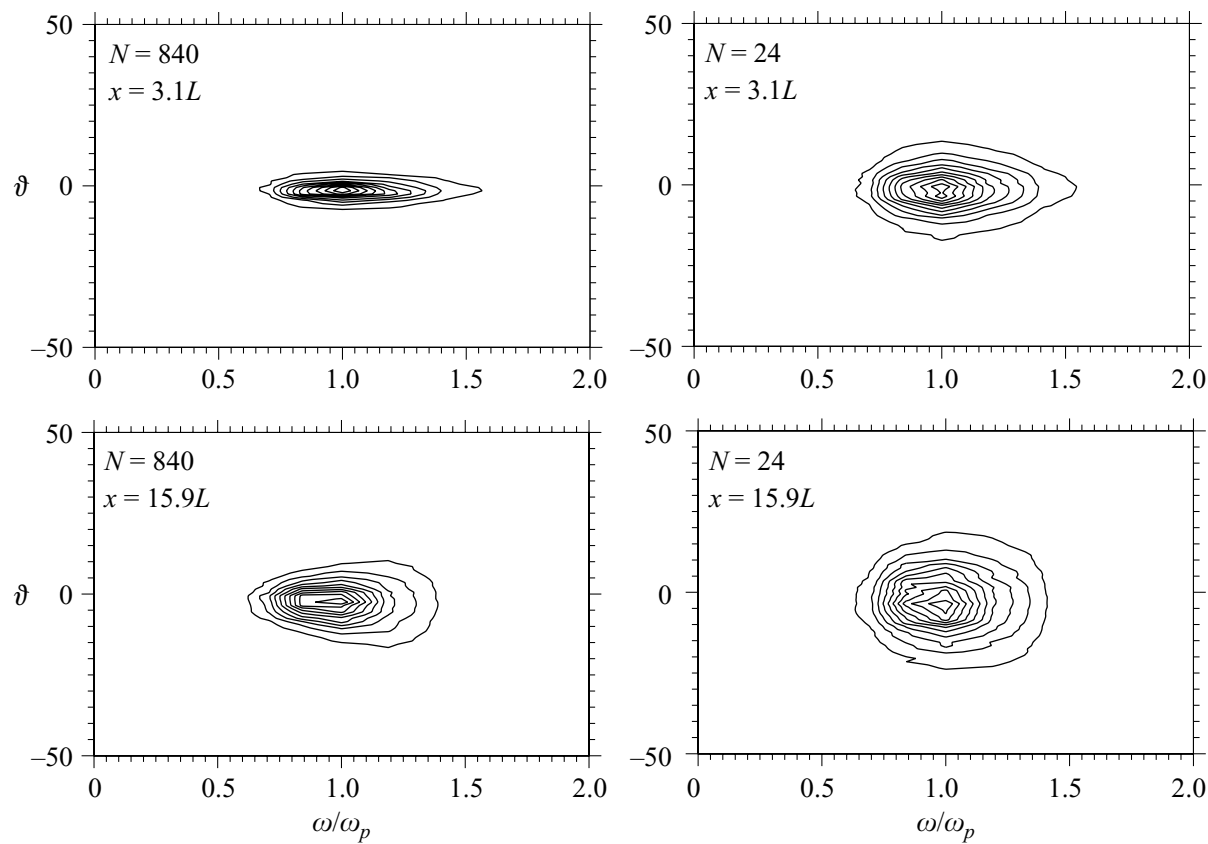

FIgURE 6. Measured directional energy spectra in a long- $(N=840)$ and short-crested $(N=24)$ wave field: at $3.1 \lambda_{p}$ (upper panels); at $15.9 \lambda_{p}$ (lower panels). 

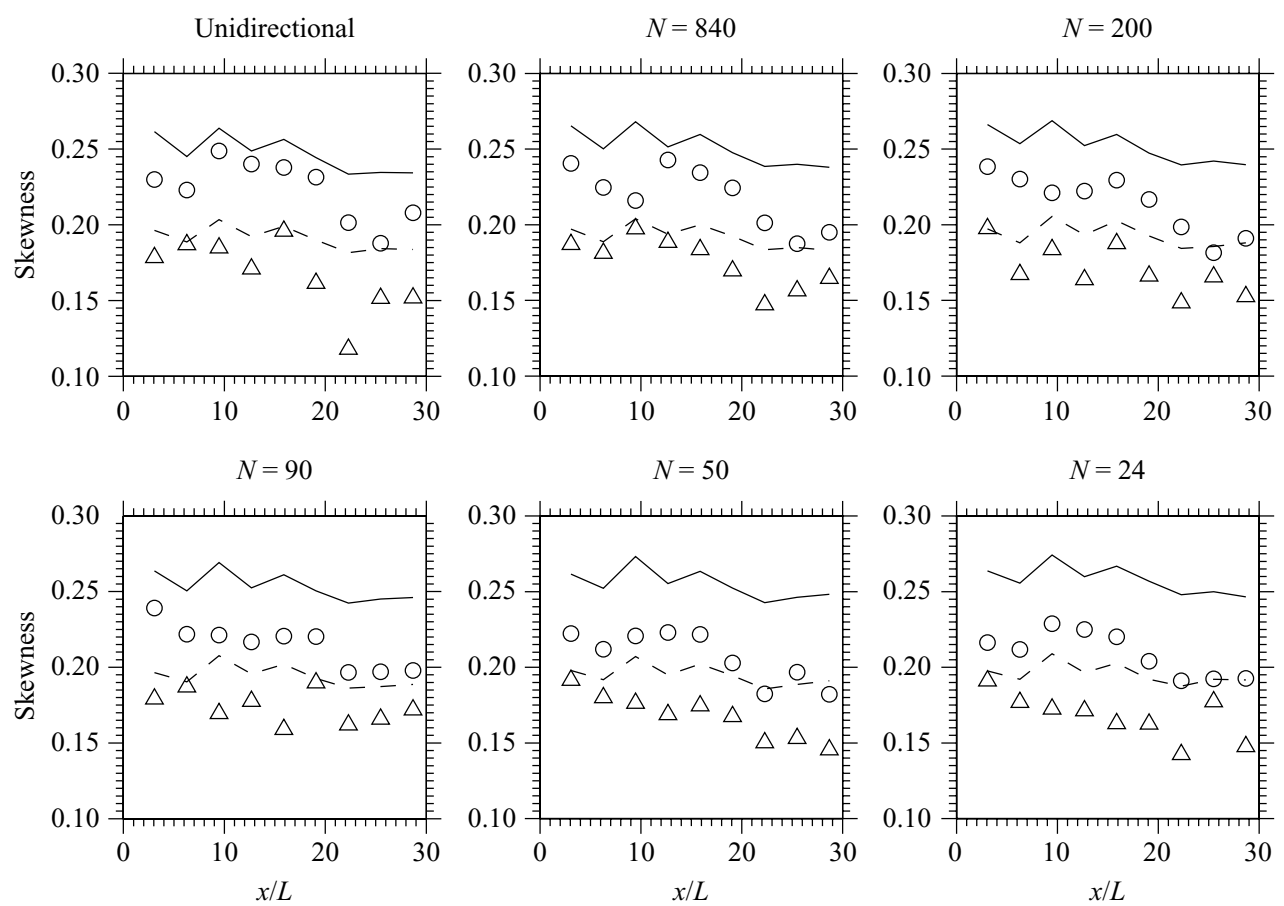

FIGURE 7. Skewness as a function of the non-dimensional distance from the wavemaker: experiment $\mathrm{A}(\triangle)$; experiment $\mathrm{B}(\mathrm{o})$; Equation (3.1) for experiment $\mathrm{A}$ (dashed line); Equation (3.1) for experiment B (solid line).
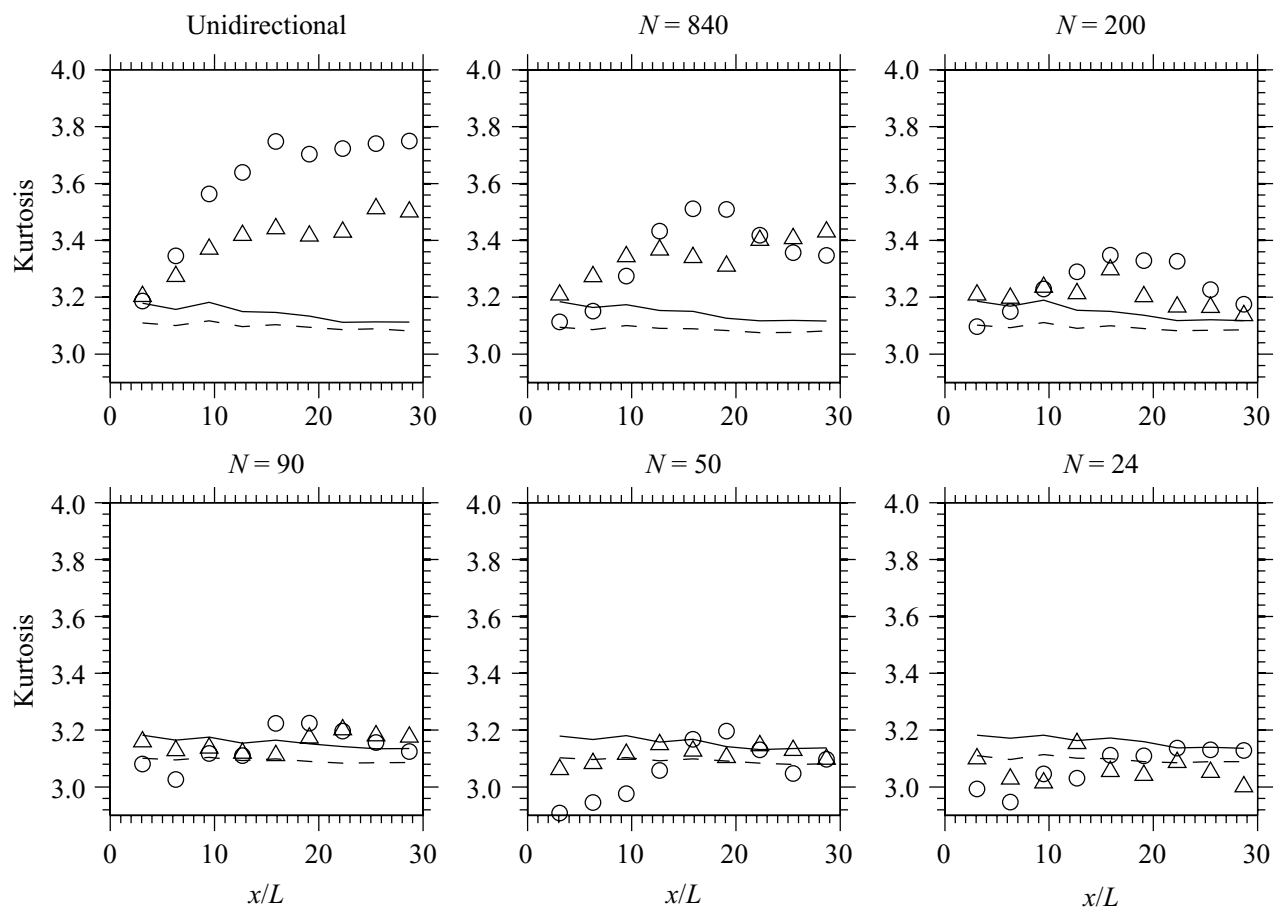

FIGURE 8. Kurtosis as a function of the non-dimensional distance from the wavemaker: experiment $A(\triangle)$; experiment $B(o)$; Equation (3.2) for experiment $A$ (dashed line); Equation (3.2) for experiment B (solid line). 
and kurtosis (see Forristall 2000; Toffoli, Onorato \& Monbaliu 2006). This is basically because in a directional spectrum the second harmonic is usually less pronounced with respect to the long-crested case (see figures 4 and 5).

For long-crested waves (i.e. unidirectional), there is a relatively good agreement between the measured skewness and (3.1), even though the latter tends to slightly overestimate the observations. There is also the tendency for the skewness to diminish as the wave field propagates along the basin. Although (3.1) shows a consistent decreasing trend, it is not able to properly capture the reduction of skewness at the end of the basin. As the initial directional spreading is enhanced the overestimation of (3.1) becomes significant at each measurement station, especially for the experiment involving steep waves $\left(k_{p} a=0.16\right)$.

Unlike the skewness, the kurtosis is more influenced by the nonlinear dynamics of free waves (see, for example, Janssen 2003; Onorato et al. 2004, 2005; Mori \& Janssen 2006), which is responsible for the formation of extreme events. In this respect, for long-crested waves, the present experiments show that the kurtosis significantly exceeds the second-order prediction (3.2) for both experiments. When directional wave fields are considered, however, the effects related to free waves is gradually reduced with the directional spreading coefficient $N$ (i.e. broad directional spreading). In particular, we observe that the contribution of free waves becomes negligible for directional spreading $N \leqslant 90$, independently from the value of the experiment. Therefore, the kurtosis results in good agreement with (3.2), which only includes the contribution of bound waves. Qualitatively, these findings are similar to previous laboratory experiments in a wave basin performed by Waseda (2006), and numerical simulations of Schödinger-type equations (Onorato et al. 2002a; Socquet-Juglard et al. 2005).

\subsection{Probability density function of the surface elevation}

In figures 9-11 we present the probability density function of the surface elevation at different distances from the wavemaker, respectively, for long-crested waves, for $N=200$ and $N=24$. For convenience, we scale the surface elevation by the standard deviation $\sigma$ of the concurrent time series. The experimental probability density function is compared to the following second-order distribution (see Socquet-Juglard et al. 2005):

$$
p(\eta)=\frac{1-7 \sigma^{2} k_{p}^{2} / 8}{\sqrt{2 \pi\left(1+3 G+2 G^{2}\right)}} \exp \left(-\frac{G^{2}}{2 \sigma^{2} k_{p}^{2}}\right)
$$

where

$$
G=\sqrt{1+2 k_{p}^{2} \sigma \eta}-1
$$

which has been derived in Socquet-Juglard et al. (2005) as an approximation of the Tayfun second-order distribution (see Tayfun 1980).

For all experiments, the experimental probability density function fits the Tayfun distribution reasonably well at the first probe $(x / L=3.1)$. The effects related to bound waves dominate the statistical properties of the wave field. As waves propagate along the basin, nevertheless, the effects related to free waves develop. For long-crested waves, this results in a significant deviation of the upper tail of the probability density function from the Tayfun distribution. These deviations are evident after about 16 wavelengths for both experiments.

For more short-crested cases $(N \leqslant 200)$, the coexistence of a number of wave components with different directions of propagation results in a significant reduction 

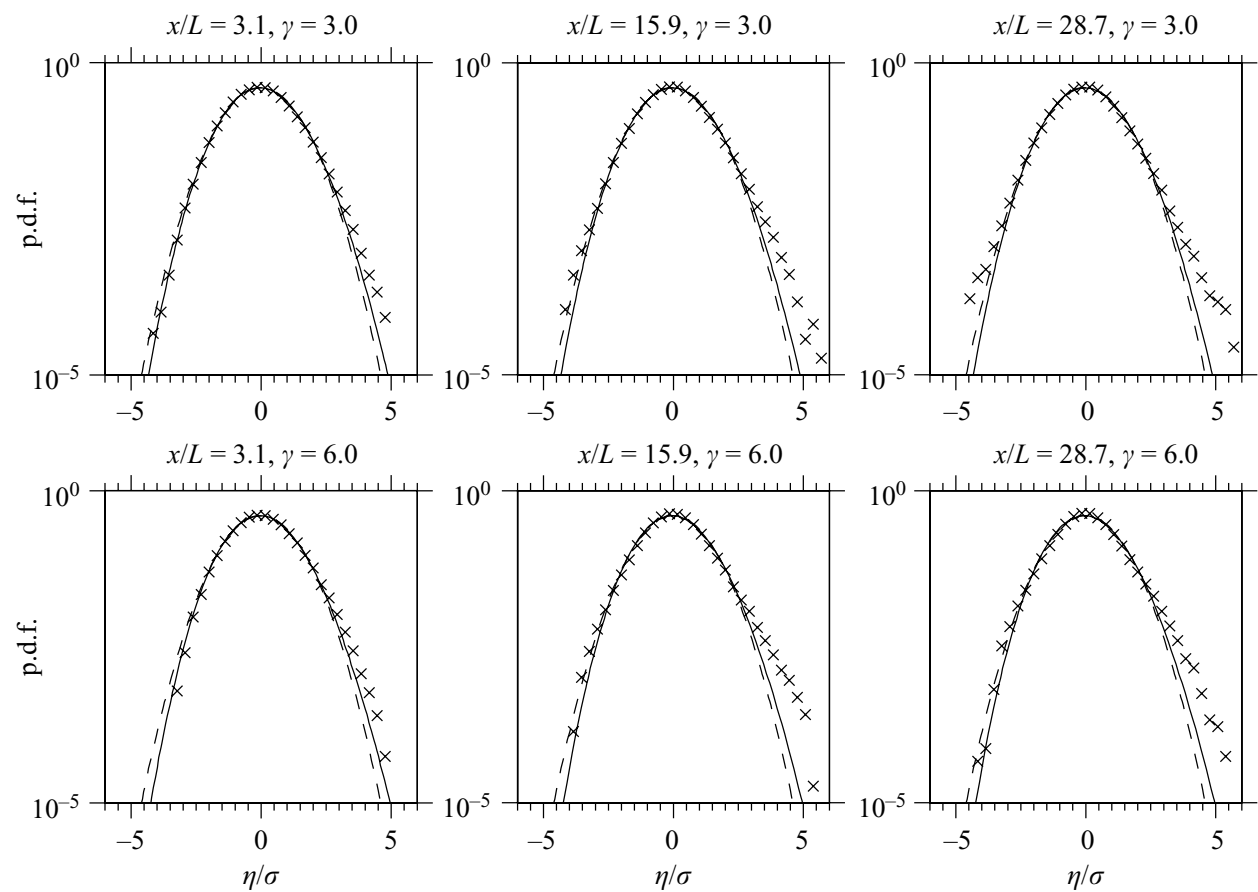

FIGURE 9. Probability density function of the surface elevations for unidirectional waves: normal distribution (dashed line); Equation (3.3) (solid line); laboratory experiments (+).
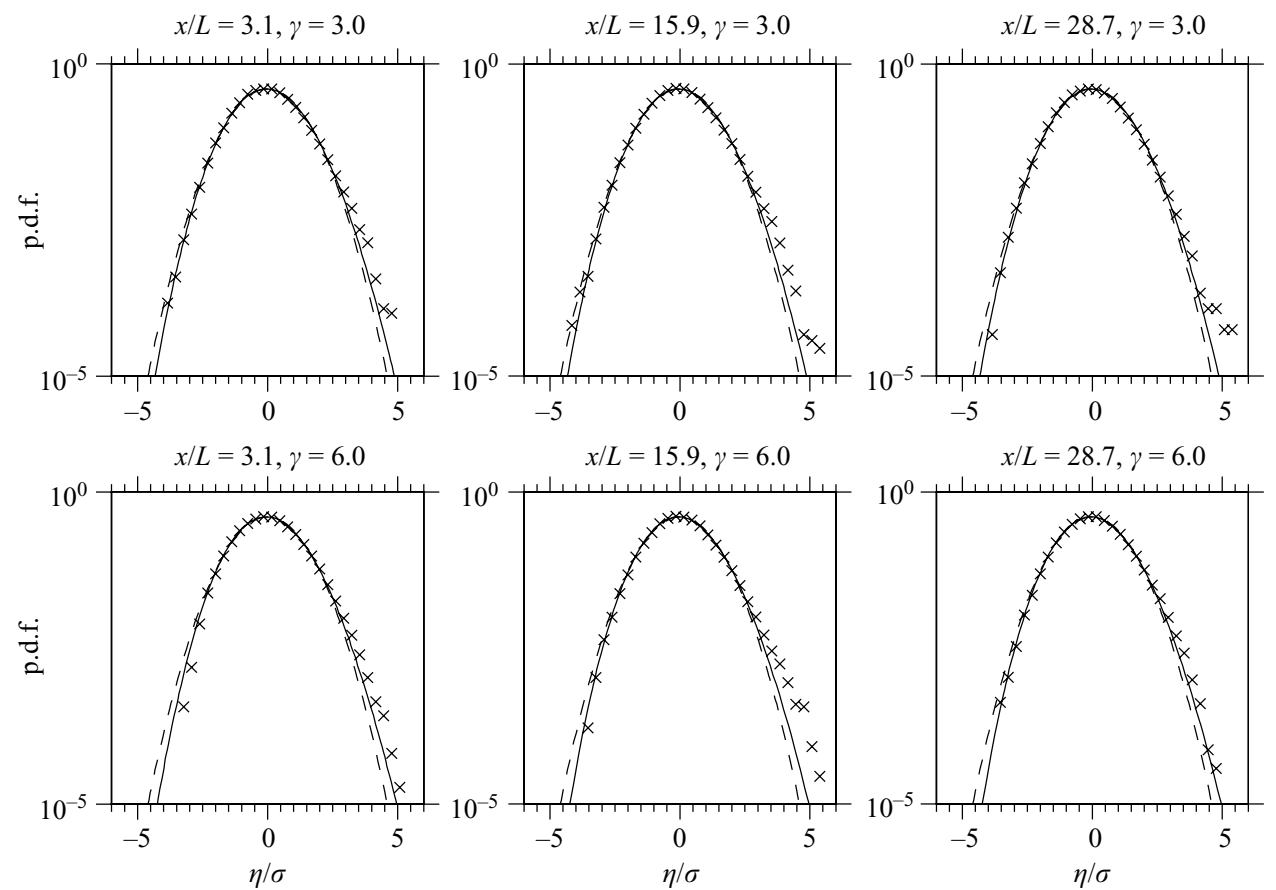

FiguRE 10. Probability density function of the surface elevations with directional spreading $N=200$ : normal distribution (dashed line); Equation (3.3) (solid line); laboratory experiments $(+)$. 

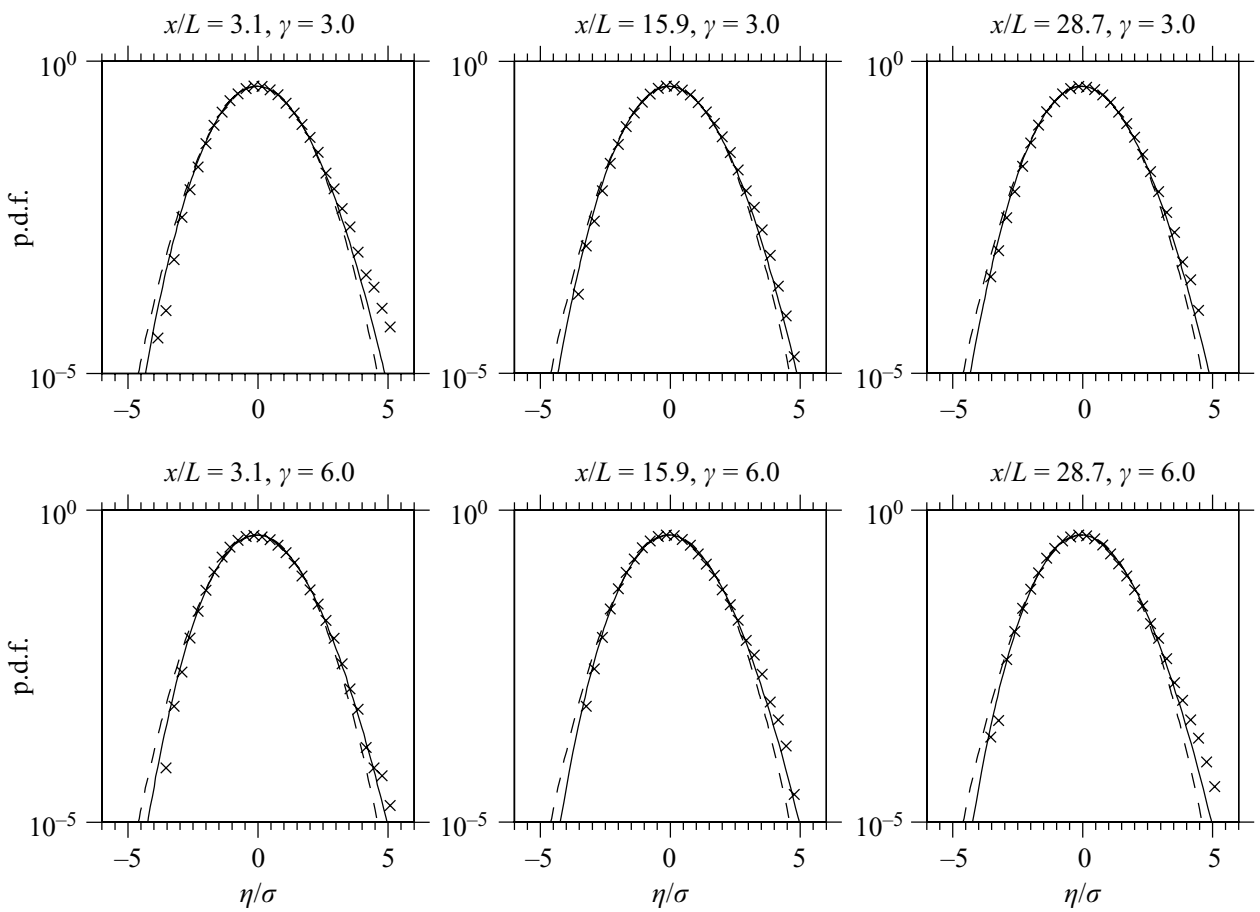

FIGURE 11. Probability density function of the surface elevations with directional spreading $N=24$ : normal distribution (dashed line); Equation (3.3) (solid line); laboratory experiments $(+)$.

of the effects of the modulational instability. As a consequence, the deviation of the tail of the probability density function from the Tayfun distribution is reduced; high crests are not more frequent than those in second-order predictions (cf. SocquetJuglard et al. 2005). Although the relevance of the effects related to the nonlinear dynamics of free long-crested waves grows with the BFI (see, e.g. Onorato et al. $2006 b$ ), large directional spreading conditions seem to suppress these effects also for large values of the $B F I$.

In order to establish the reliability of the present experimental results, we show in figure 12 the error bars for the upper tail of the probability density function for long-crested waves, for $N=200$ and for $N=24$. The error bars are computed as $\pm p(\eta) / \sqrt{N_{b}}$, where $N_{b}$ is the number of events in each bin. Although the confidence interval increases with the decrease of the probability levels, estimates of the probability density functions are accurate for probability levels as low as 0.0001 .

\subsection{Wave height}

Here we investigate the exceedance probability of the wave height calculated as the zero-up and the zero-down crossing waves. The evolution of the experimental distribution along the wave basin is presented in figures 13-15, respectively, for long-crested waves, for $N=200$ and for $N=24$. The wave height is normalized using significant wave height estimated as four times the standard deviation of the concurrent time series. Furthermore, we expect that the second-order theory should not affect the wave height distribution, which, according to linear theory, should be very close to the Rayleigh distribution. The latter, therefore, is used as a reference distribution. 


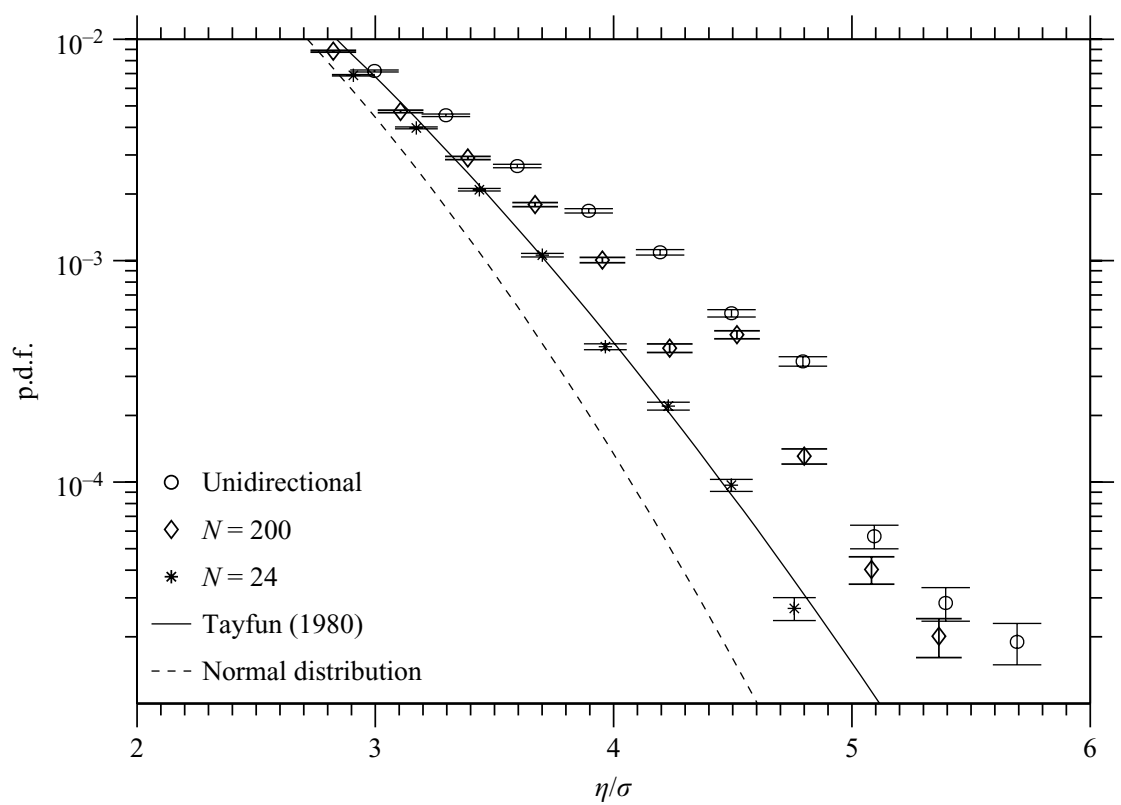

FIGURE 12. Upper tail of the probability density function of the surface elevations.

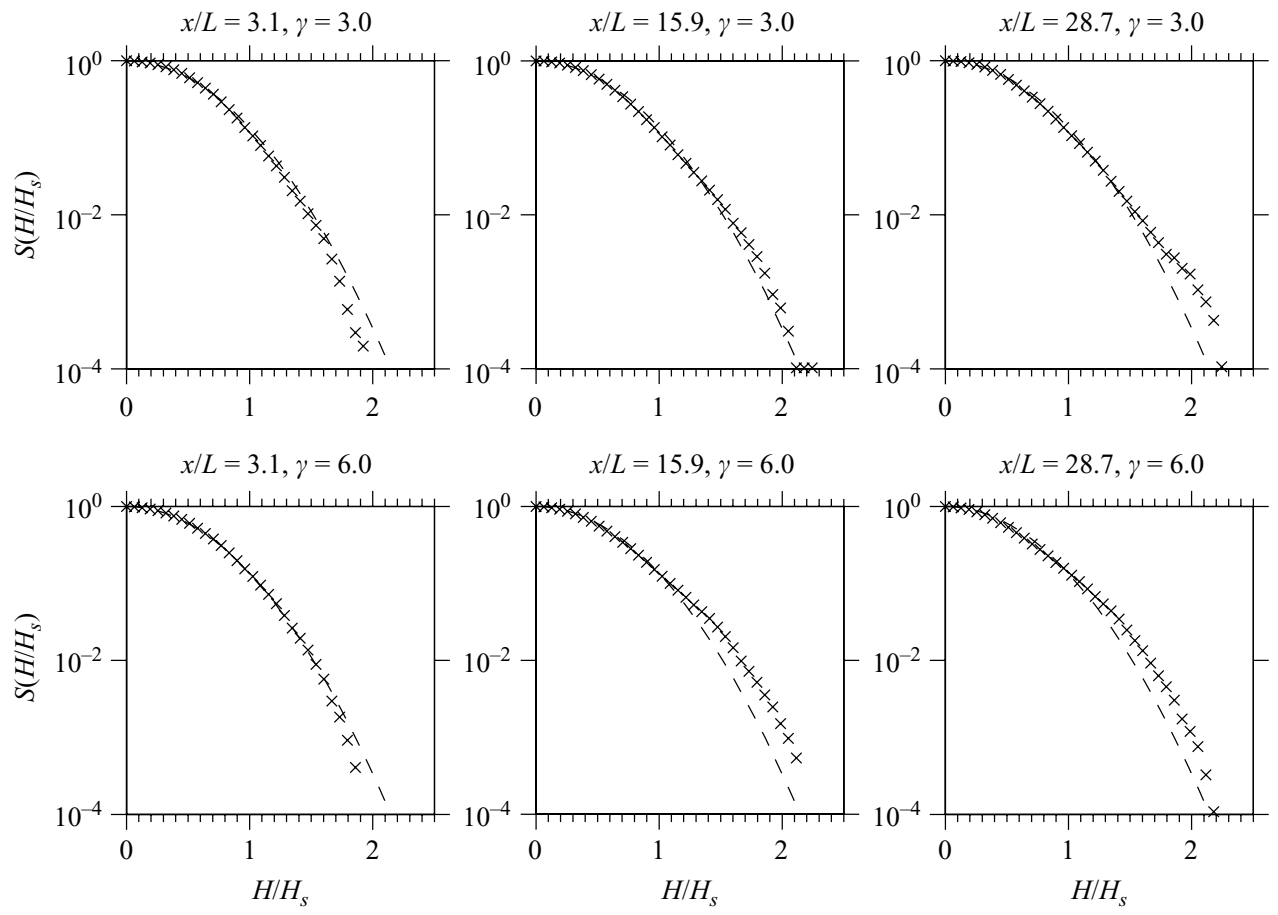

FIGURE 13. Exceedance probability of the wave height for unidirectional sea states: Rayleigh distribution (dashed line); laboratory experiments $(\times)$.

At the first probe $(x / L=3.1)$, the Rayleigh distribution describes relatively well the experimental data down to probability levels of 0.001 ; for lower probabilities, the Rayleigh distribution slightly overestimates the measurements (see Longuet-Higgins 

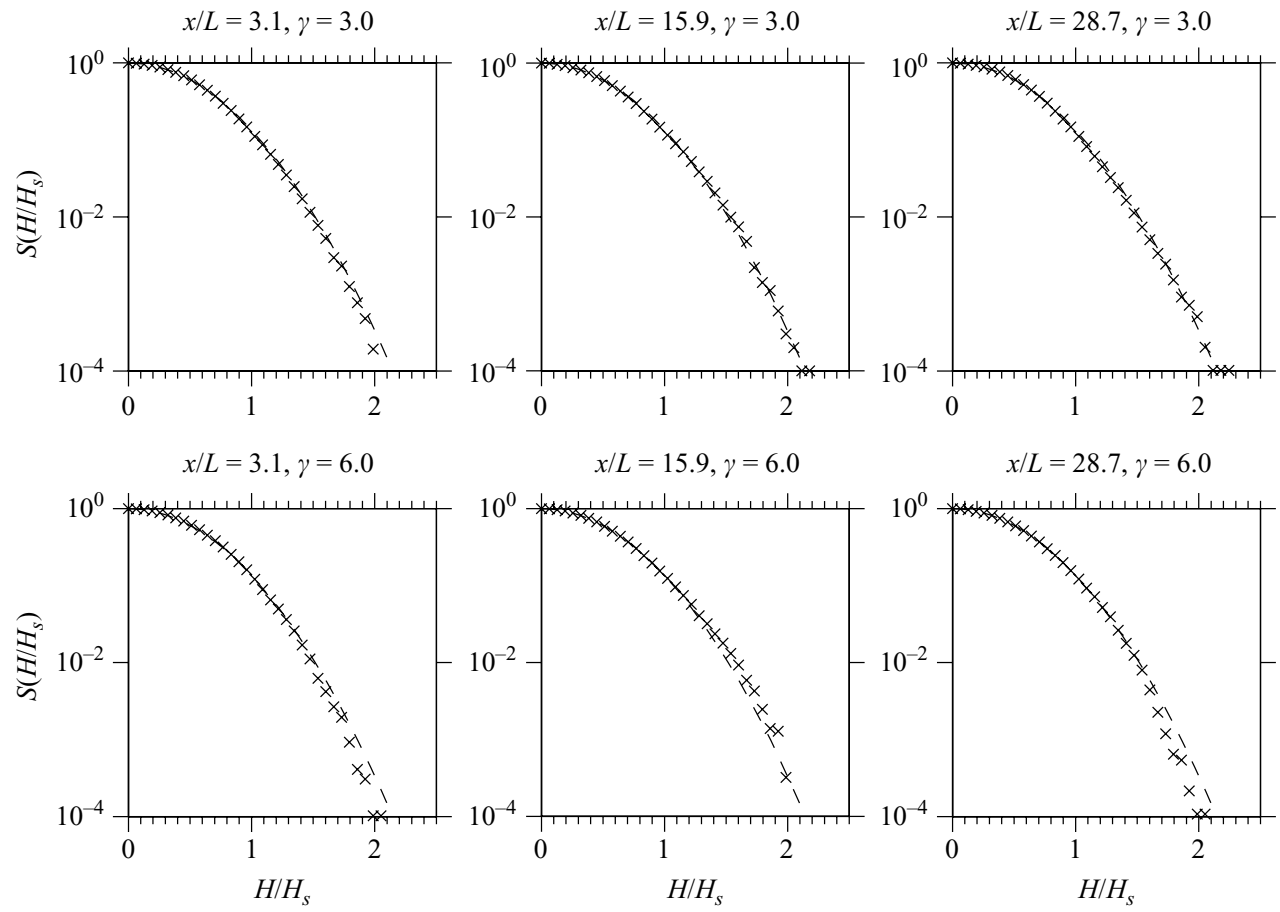

FIGURE 14. Exceedance probability of the wave height for directional sea states with directional spreading $N=200$ : Rayleigh distribution (dashed line); laboratory experiments $(\times)$.
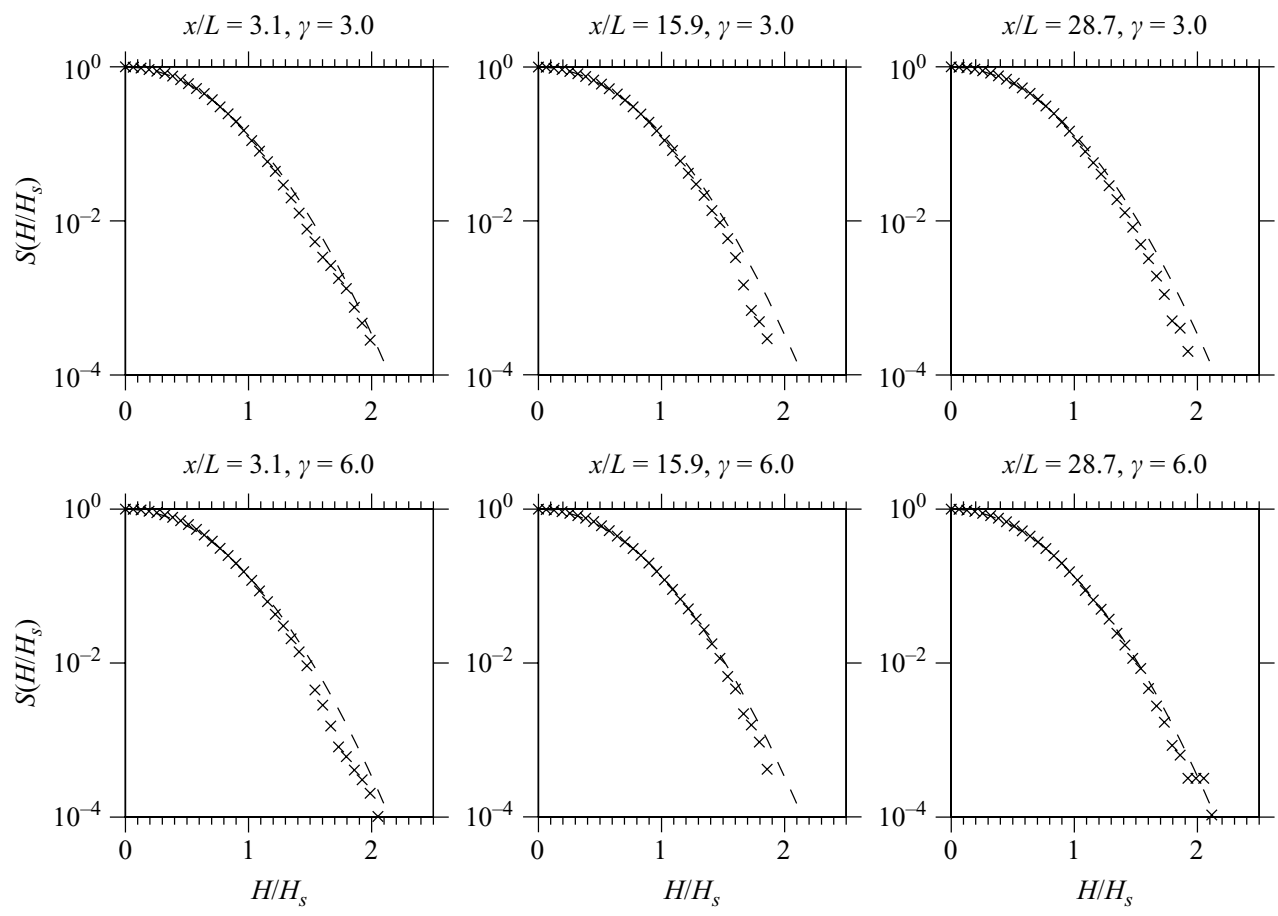

FIGURE 15. Exceedance probability of the wave height for directional sea states with directional spreading $N=24$ : Rayleigh distribution (dashed line); laboratory experiments $(\times)$. 
1980 for a discussion). We recall, in this respect, that the wave field has been generated at the wavemaker as a linear superposition of random waves. For unidirectional waves, the modulational instability produces large wave heights, which modify the wave height distribution already after about 16 wavelengths. As expected, this deviation is more evident for experiment B. Previous flume experiments performed by Onorato et al. (2006b) have shown that this departure starts at probability levels as low as 0.1 . For the present experiments, however, the deviation from the Rayleigh distribution is observed at probability levels one order of magnitude lower (i.e. 0.01). This may be related to the fact that, unlike in a narrow wave flume, long-crested waves do not persist along the basin.

As the directional spreading is introduced at the wavemaker, it appears that the Rayleigh distribution provides an appropriate description of the measured wave heights. Here we emphasize that, even for a Gaussian wave field (but of finite bandwidth), the wave height distribution is only approximately of the Rayleigh type. The fact that the wave height distribution for the observed waves reduces to a Rayleigh distribution does not imply that the wave field is fully Gaussian; it could be the result of compensating deviations from non-Gaussian and finite-bandwidth effects.

Although the time series were recorded with very high sampling frequency, the number of individual waves available for the analysis was relatively low (about 8000 waves). Therefore, the distribution at low probability levels may be uncertain. However, the analysis of the $95 \%$ confidence interval performed with bootstrap methods (see, for example Emery \& Thomson 2001) showed that the estimate of the wave height distribution is accurate at probability levels as low as 0.001 .

\subsection{Wave crest}

We now consider the statistical distribution of wave crests, which are defined as the highest elevation of each individual wave with respect to the mean water level. For the crest amplitude, the second-order interactions should participate to the deviation from Gaussian statistics. Under the hypothesis of deep water and narrow-banded waves, Tayfun (1980) has derived a second-order wave crest distribution. The exceedance probability assumes the following form:

$$
S(C>\eta)=\exp \left[-\frac{8}{H_{s}^{2} k_{p}^{2}}\left(\sqrt{1+2 k_{p} \eta}-1\right)^{2}\right],
$$

where $C$ is the crest height and $k_{p}$ is the wavenumber of the dominant wavelength. In figures 16-18, the second-order wave crest distribution is compared with the laboratory experiments.

At probes nearby the wavemaker $(x / L=3.1)$, the second-order theory provides a good estimate of the experimental data. As waves propagates along the basin, the effects related to the nonlinear dynamics of free waves dominate the statistical properties of the wave crests leading to a substantial deviation from the Tayfun distribution (3.5), provided waves are long crested (see also Onorato et al. 2006b). Towards the end of the basin, nevertheless, the crest amplitude attenuates and so the deviation from second-order theory. Similar results were also obtained numerically by Socquet-Juglard et al. (2005). As mentioned for the wave height distribution, also the estimate of the wave crest distribution results to be rather accurate for probability levels as low as 0.001 (within $95 \%$ confidence interval).

As the directional distribution is increased (waves become more short crested), the deviation from the second-order theory is reduced. For large directional spreading 


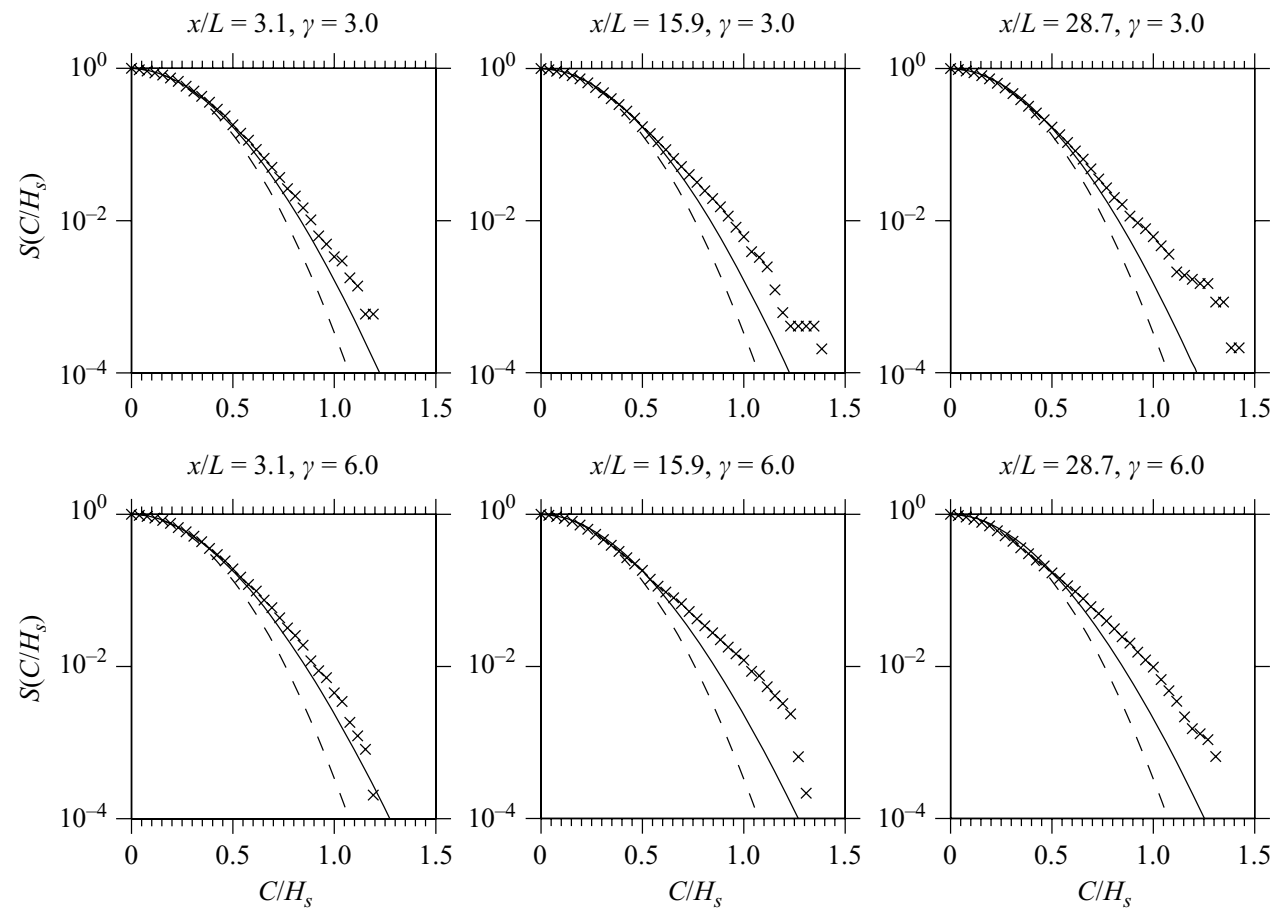

FIGURE 16. Exceedance probability of the wave crest for unidirectional sea states: Rayleigh distribution (dashed line); Equation (3.5) (solid line); laboratory experiments $(\times)$.

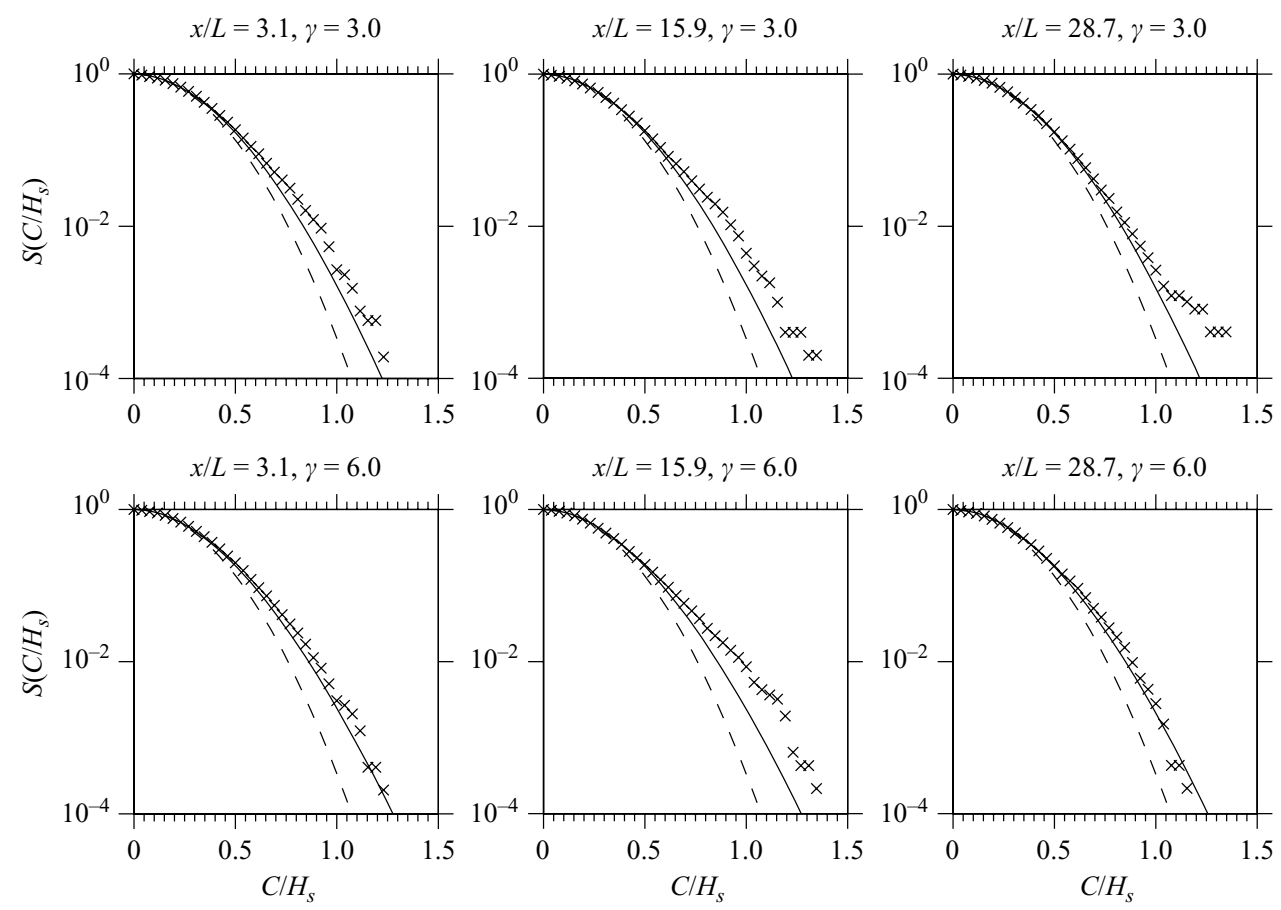

FIGURE 17. Exceedance probability of the wave crest for directional sea states with directional spreading $N=200$ : Rayleigh distribution (dashed line); Equation (3.5) (solid line); laboratory experiments $(\times)$. 

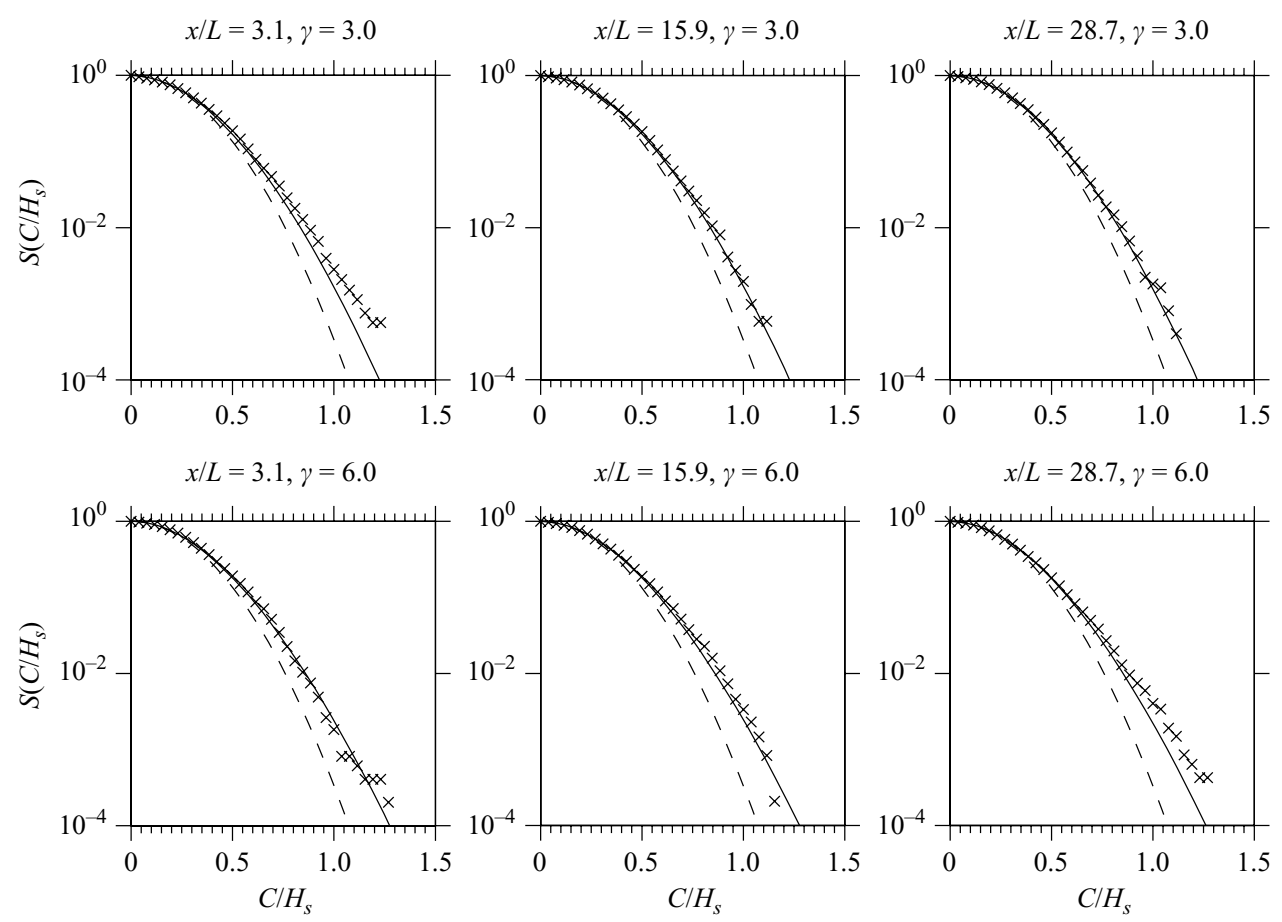

FIGURE 18. Exceedance probability of the wave crest for directional sea states with directional spreading $N=24$ : Rayleigh distribution (dashed line); Equation (3.5) (solid line); laboratory experiments $(x)$.

(3.5) well approximates the experimental data. In such sea states, therefore, the effects related to bound waves (second-order theory) dominate the statistical properties of the crest amplitude (cf. Socquet-Juglard et al. 2005).

\section{Discussion and conclusions}

In the previous sections, we have presented a detailed analysis of some of the statistical properties of the surface elevation. The skewness, the kurtosis, the probability density function for surface elevation, for wave heights and for wave crests have been presented as a function of the distance from the wavemaker for experiments $\mathrm{A}$ and $\mathrm{B}$, for different directional spreadings, ranging from long- to short-crested wave fields. In order to summarize the results, here we present some key figures, which highlight the relevance of the directional distribution on wave statistics. For convenience, we shall concentrate on the most nonlinear case considered, i.e. experiment $\mathrm{B}$.

In figure 19, we present the kurtosis as a function of the distance from the wavemaker for different values of the spreading coefficient $N$. For narrow directional distribution, as waves propagate along the basin, the kurtosis increases, deviating from Gaussian statistics; it grows for about 15-20 wavelengths (middle of the basin), where it reaches its maximum. It is evident that departures from Gaussian statistics are particularly significant if waves are fairly long crested. For more short-crested conditions (small $N$ ), extreme waves occur less often. Therefore, after an initial growth, the kurtosis reaches values which do not exceed the second-order prediction. This 


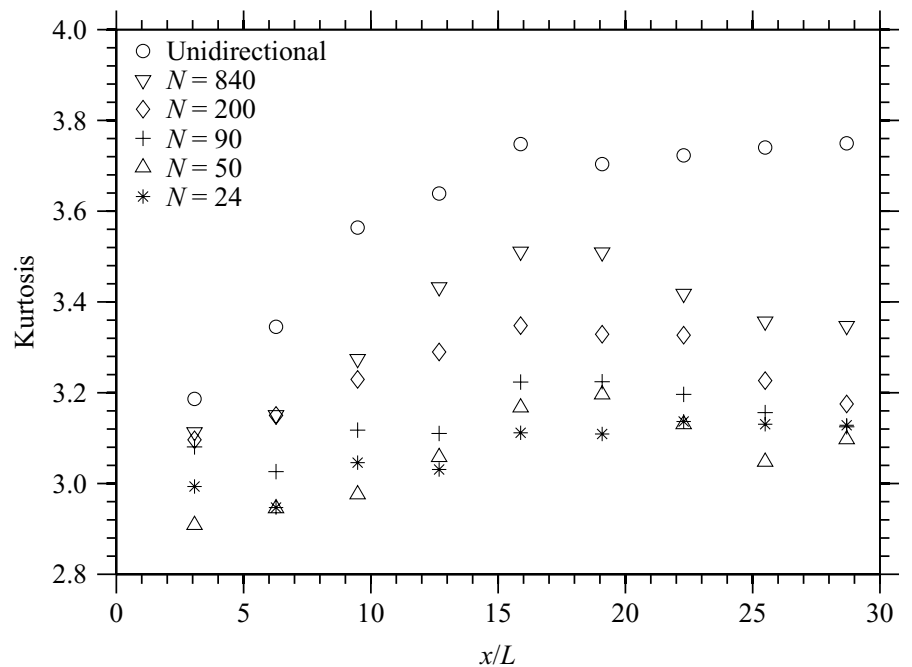

FIGURE 19. Kurtosis as a function of distance from the wavemaker for different values of $N$ for experiment $\mathrm{B}$.

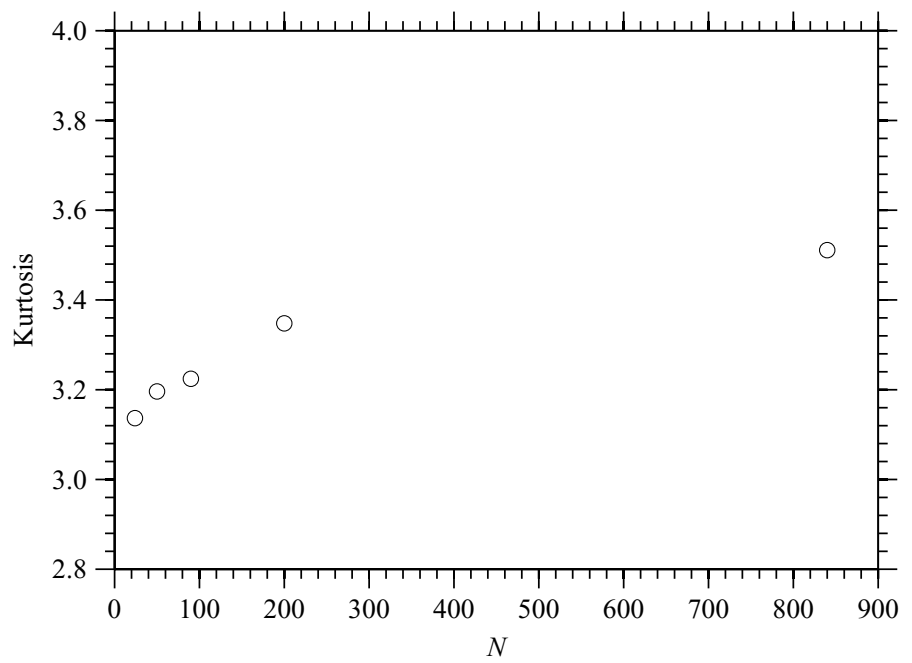

Figure 20. Maxima of kurtosis as a function of $N$ for experiment B.

is clearly summarized in figure 20, where the maximum values of the kurtosis are shown as a function of the directional spreading. It is clear that the maximum of the kurtosis grows with $N$, i.e. it increases as waves become long crested.

In figure 21 we show the probability density functions of the surface elevation estimated from the time series recorded at the probe where the kurtosis reaches its maximum and compare them for different values of $N$. This figure shows two important results: (i) for quasi-long-crested waves, the appearance of extreme waves can be underestimated by about one order of magnitude if second-order theory is considered; (ii) for large directional distribution, the probability of occurrence of extremes is well described by second-order theory.

The appearance of deviations from second-order theory is possibly due to the modulational instability process which is a quasi-resonant process that takes place 


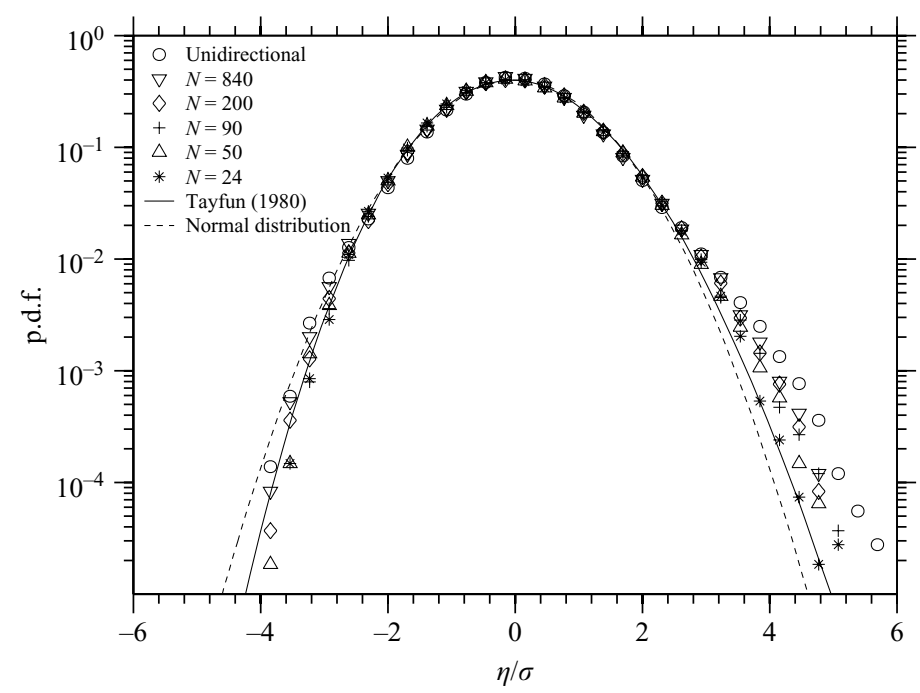

FIGURE 21. Probability density function of the surface elevation at the probe of maximum kurtosis for experiment $\mathrm{B}$.

around the peak of the spectrum. At this point a short digression on the directional dependence of the energy spectrum for ocean waves is needed to better understand the relevance of the aforementioned results. If ocean spectra are always broad banded, i.e. characterized by small $N$, the results here obtained for large $N$ are interesting on their own but probably are not very relevant for ocean waves. Thus, a main question that must be answered is the following: what is the directional distribution of the energy spectrum around the spectral peak? Before entering this discussion, we recall here that the directional properties of the surface elevation are usually achieved with in-situ measurements using pitch and roll buoys or array of sensors. Young (1994) studied in detail different parametrizations of directional spectra available in the literature. In figure 2 of his paper he compares the three different available parametrizations of the directional spreading (Mitsuyasu et al. 1975; Hasselmann, Dunckel \& Ewing 1980; Donelan, Hamilton \& Hui 1985); the figure clearly shows that there is not a decent agreement between the three of them. He also considered two different methods that are usually adopted to recover the directional information: the Fourier Expansion Method and the Maximum Likelihood Method. After selecting a nominal (input) spectrum, he numerically simulated the output of a pitch and roll buoy (or an array composed by three gauges). He then used the aforementioned methods to estimate the directional properties of the wave spectrum. He concluded that (Young 1994, p. 288): 'Both analysis techniques produce directional spreading which is too broad and significantly underestimate the magnitude of the narrow directional distribution'. We notice that the nominal spectrum that he considered in his analysis is not so narrow, thus we expect that the effect could become even more pronounced for narrow spectra. According to Young (1994), an improvement of the accuracy of the estimation of the directional spectrum can be achieved only if the number of sensor is increased. Nonetheless, even with an array of seven gauges, his results show that the energy at the peak of the spectrum is underestimated by $30 \%$. In Donelan et al. (1996) a method based on the wavelet transform for estimating the directional wave spectrum has been developed. The method seems promising and capable of reproducing accurately the width in angle of the spectrum at the peak 
frequency; nevertheless, to our knowledge, no parametrization of the directional wave spectrum has been attempted using such method.

Concerning remote-sensing methods, Hwang et al. (2000) have used airborne scanning lidar measurements of wavenumber spectra of ocean surface waves to investigate their directional properties. Although, the resolution in angle increases with wavenumbers, it is $10^{\circ}$ at the peak of the spectrum; unfortunately, this resolution is still poor for detecting accurately the width of the spectrum at the peak. Therefore, it is reasonable to assume that no confident results on the directional distribution of energy at the peak are available. Hence, it cannot be excluded a priori that some natural conditions could exist in which the directional distribution is much smaller than estimated from experimental data. This condition is clearly a candidate for extreme waves. In this respect, new research is called for. It should also be emphasized that the results herein discussed only concern single-peaked wave spectra. The case of two peaks (for example the interaction of swell and wind sea) still has to be addressed properly in terms of the modulational instability (for a first preliminary work see Onorato, Osborne \& Serio 2006a). This is also a subject that should be investigated further.

It should also be mentioned here that the effect of wind has not been considered in the present experimental investigation. Surely, the wind is relevant in order to sustain the peak of the spectrum but its direct effect on the formation of extreme waves and on the modulational instability in random waves is an issue to be explored. For linear focusing, some experiments have been performed in a wave flume by Kharif et al. (2008). Their results suggest that extreme wave events may be sustained longer by the air flow separation occurring on the leeward side of the steep crests.

To conclude, we have presented a very detailed description of the statistical properties of surface gravity waves in different conditions of nonlinearity and directional spreading. Apart from the very recent work of Waseda (2006), we are not aware of any other study in which the probability density function of the surface elevation has been investigated with special attention to the role of the angular distribution. The modulational instability process, which is one of the main mechanisms of formation of extreme waves in deep water random long-crested waves, seems to be quenched when short-crested waves are considered. We believe that, after many years of numerical and theoretical research, this represents an important step towards the understanding of the physics of extreme waves. At the moment, the lack of an accurate estimation of the peak angular spectral energy distribution for different sea state conditions represents a gap to be filled up soon. We do not expect the directional distribution to be very different from what is already known by now, nonetheless the peak of the spectrum does not seem to be well resolved in any experiment we are aware of. We suspect that, as there exists an enhancement factor (the $\gamma$ in the JONSWAP spectrum) for the frequency spectrum, there could also exist a similar factor in the angular part of the spectrum. If we accept the idea that the $\gamma$ is the result of a resonance in the generation mechanism, why would the wind generate a narrow spectrum in frequency at the peak and a broad one in angle? It should also be mentioned that recently a direct connection between the breaking phenomena and the modulational instability has been discussed in Song \& Banner (2002), therefore we believe that the present results may also represent a first step towards the development of a new dissipation source term in the energy balance equation.

We are aware of the fact that waves in the basin are generated artificially and that their evolution is related to the rather special boundary conditions. However, in nature, strong wind, rapid changes in the wind, currents and refraction could 
in principle reproduce the condition of a narrow spectrum also in angle. If such conditions are in nature reproduced, then it could be possible to associate a value of the kurtosis to each spectrum, and from it, using the approach described in Mori \& Janssen (2006), an estimation of the probability of extreme wave could be associated.

The experimental work was supported by the European Community's Sixth Framework Programme through the grant to the budget of the Integrated Infrastructure Initiative HYDROLAB III, Contract no. 022441 (RII3). We thank D. Resio, N. Mori and $\mathrm{T}$. Waseda for valuable discussions.

\section{REFERENCES}

Alber, I. E. 1978 The effects of randomness on the stability of two dimensional surface wave trains. Proc. R. Soc. Lond. A 636, 525-546.

Benjamin, T. B. \& Feir, J. E. 1967 The disintegration of wave trains on deep water. J. Fluid Mech. 27, 417-430.

BRANDINI, C. 2001 Nonlinear interaction processes in extreme wave dynamics. PhD thesis, Universitá Di Firenze.

Denissenko, P., Lukaschuk, S. \& Nazarenko, S. 2007 Gravity wave turbulence in a laboratory flume. Phys. Rev. Lett. 99, 014501.

Donelan, M. A., Drennan, W. M. \& Magnusson, A. K. 1996 Nonstationary analysis of the directional properties of propagating waves. J. Phys. Oceanogr. 26, 1901-1914.

Donelan, M. A., Hamilton, J. \& Hui, W. H. 1985 Directional spectra pf wind-generated waves. Philos. Trans. R. Soc. Lond. A 315, 509-562.

Dysthe, K. B. 1979 Note on the modification of the nonlinear Schrödinger equation for application to deep water waves. Proc. R. Soc. Lond. A369, 105-114.

Dysthe, K. B., Trulsen, K., Krogstad, H. \& Socquet-Juglard, H. 2003 Evolution of a narrowband spectrum of random surface gravity waves. J. Fluid Mech. 478, 1-10.

Emery, W. J. \& Thomson, R. E. 2001 Data Analysis Methods in Physical Oceanography. Elsevier Science B.V.

Forristall, G. Z. 2000 Wave crests distributions: Observations and second-order theory. J. Phys. Oceanogr. 30, 1931-1943.

Gramstad, O. \& Trulsen, K. 2007 Influence of crest and group length on the occurrence of freak waves. J. Fluid Mech. 582, 463-472.

Hasselmann, K. 1962 On the non-linear energy transfer in a gravity-wave spectrum. Part I. General theory. J. Fluid Mech. 12, 481-500.

Hasselmann, D. E., Dunckel, M. \& Ewing, J. A. 1980 Directional wave spectra observed during jonswap 1973. J. Phys. Oceanogr. 10, 1264-1280.

Hauser, D., Kahma, K. K., Krogstad, H. E., Lehner, S., Monbaliu, J. \& Wyatt, L. W. (Ed.) 2005 Measuring and Analysing the Directional Spectrum of Ocean Waves. Cost Office.

Hwang, P. A., Wang, D. W., Walsh, E. J., Krabill, W. B. \& Swift, R. N 2000 Airborne measurements of the wavenumber spectra of ocean surface waves. Part II. Directional distribution. J. Phys. Oceanogr. 30, 2768-2787.

Janssen, P. A. E. M. 2003 Nonlinear four-wave interaction and freak waves. J. Phys. Oceanogr. 33 (4), 863-884.

Janssen, P. A. E. M. \& Onorato, M. 2007 The intermediate water depth limit of the Zakharov equation and consequences for wave prediction. J. Phys. Oceanogr. 37, 2389-2400.

Kharif, C., Giovanangeli, J. P., Touboul, J., Grare, L. \& Pelinovsky, E. 2008 Influence of wind on extreme wave events: experimental and numerical approaches. J. Fluid Mech. 594, 209247.

Komen, G. J., Cavaleri, L., Donelan, M., Hasselmann, K., Hasselmann, H. \& Janssen, P. A. E. M. 1994 Dynamics and Modeling of Ocean Waves. Cambridge University Press.

Longuet-Higgins, M. S. 1963 The effect of non-linearities on statistical distribution in the theory of sea waves. J. Fluid Mech. 17, 459-480. 
Longuet-Higgins, M. S. 1980 On the distribution of the heights of sea waves: some effects of nonlinearity and finite band width. J. Geophys. Res. 85, 1519-1523.

Mitsuyasu, H., Tasai, F., Suhara, T., Mizuno, S., Ohkusu, M., Honda, T. \& Rikishis, K. 1975 Observations of the directional spectrum of ocean waves using a cloverleaf buoy. J. Phys. Oceanogr. 5, 750-760.

Mori, N. \& JANSSEN, P. A. E. M. 2006 On kurtosis and occurrence probability of freak waves. J. Phys. Oceanogr. 36, 1471-1483.

Mori, N., Onorato, M., Janssen, P. A. E. M., Osborne, A. R. \& Serio, M. 2007 On the extreme statistics of long-crested deep water waves: theory and experiments. J. Geophys. Res. 112 (C9), C09011.

Mori, N. \& YASUdA, T. 2002 Effects of high-order nonlinear interactions on unidirectional wave trains. Ocean Engng 29, 1233-1245.

Onorato, M., Osborne, A. R. \& Serio, M. $2006 a$ Modulation instability in crossing sea states: A possible machanism for the formation of freak waves. Phys. Rev. Lett. 96, 014503.

Onorato, M., Osborne, A. R. \& Serio, M. $2002 a$ Extreme wave events in directional random oceanic sea states. Phys. Fluids 14 (4), 25-28.

Onorato, M., Osborne, A. R., Serio, M. \& Bertone, S. 2001 Freak wave in random oceanic sea states. Phys. Rev. Lett. 86 (25), 5831-5834.

Onorato, M., Osborne, A. R., Serio, M. \& Cavaleri, L. 2005 Modulational instability and non-gaussian statistics in experimental random water-wave trains. Phys. Fluids 17, 078101-4.

Onorato, M., Osborne, A. R., Serio, M., Cavaleri, L., Brandini, C. \& Stansberg, C. T. 2004 Observation of strongly non-gaussian statistics for random sea surface gravity waves in wave flume experiments. Phys. Rev. E 70, 067302.

Onorato, M., Osborne, A., Serio, M., Cavaleri, L., Brandini, C. \& Stansberg, C. T. $2006 b$ Extreme waves, modulational instability and second order theory: wave flume experiments on irregular waves. Eur. J. Mech. B/Fluids 25, 586-601.

Onorato, M., Osborne, A. R., Serio, M., Resio, D., Puskarev, A., Zakharov, V. E. \& Brandini, C. $2002 b$ Freely decaying weak turbulence for sea surface gravity waves. Phys. Rev. Lett. 89, 4.144501 .

Socquet-Juglard, H., Dysthe, K., Trulsen, K., Krogstad, H. E. \& Liu, J. 2005 Distribution of surface gravity waves during spectral changes. J. Fluid Mech. 542, 195-216.

Song, J. \& BANNER, M. L. 2002 On determining the onset and strength of breaking for deep water waves. Part I. Unforced irrotational wave groups. J. Phys. Oceanogr. 32, 2541-2558.

StansberG, C. T. 1992 On spectral instabilities and development of nonlinearities in propagating deep-water wave trains. In Coastal Engineering, Proceedings of the XXIII International Conference Venice, Italy, pp. 658-671. American Society of Civil Engineers.

StansBerg, C. T. 1994 Effects from directionality and spectral bandwidth on non-linear spatial modulations of deep-water surface gravity waves. In Proceedings, Vol. 1, the 24th International Conference on Coastal Engineering, Kobe, Japan, pp. 579-593. American Society of Civil Engineers.

TAYFun, M. A. 1980 Narrow-band nonlinear sea waves. J. Geophys. Res. 85 (C3), 1548-1552.

TAYfun, M. A. \& Fedele, F. 2007 Wave-height distributions and nonlinear effects. Ocean Engng 34, $1631-1649$.

TAYFun, M. A. \& Lo, J.-M. 1990 Nonlinear effects on wave envelope and phase. J. Water. Port Coastal Ocean Engng, ASCE 116, 79-100.

Toffoli, A., Onorato, M. \& Monbaliu, J. 2006 Wave statistics in unimodal and bimodal seas from a second-order model. Eur. J. Mech. B/Fluids 25, 649-661.

Trulsen, K. \& Dysthe, K. B. 1997 Freak waves - a three-dimensional wave simulation. In Proceedings of the 21st Symposium on Naval Hydrodynamics, Washington, DC, pp. 550-560. National Academy Press.

WASEDA, T. 2006 Impact of directionality on the extreme wave occurrence in a discrete random wave system. In Proceedings of 9th International Workshop on Wave Hindcasting and Forecasting, Victoria, Canada.

YounG, I. R. 1994 On the measurement of directional wave spectra. Appl. Ocean Res. 16, 283-294.

Zakharov, V. 1968 Stability of period waves of finite amplitude on surface of a deep fluid. J. Appl. Mech. Tech. Phys. 9, 190-194. 